\title{
Active Noise Cancellation: The Unwanted Signal and the Hybrid Solution
}

\author{
Edgar Omar López-Caudana \\ Tecnológico de Monterrey, Campus Ciudad de México \\ Mexico
}

\section{Introduction}

The need to eliminate unwanted sounds is greater, as this is an expression of the limited tolerance that we have as individuals to the perception of sounds generated by industrial equipment, appliances and some general properties that are unpleasant for most people. Vibrations mechanical engines in operation, digging machinery and electricity generators, are just a few examples of processes or equipments that can produce signals nuisance to human ear.

While mitigating these unwanted sounds already exists today, the methods used are based on passive elements that offer a poor response to low frequency sounds. This drawback happens (Kuo \& Morgan, 1996), when the wavelength of the signal is long compared to the size of the muffler liabilities. The relevance in the treatment of low-frequency sounds is that they produce fatigue and loss of concentration, thus affecting the performance of people, machinery and equipment present. That is, low-frequency sounds produced very intense vibrations that can fracture structures in the team very long periods of exposure.

ANC Systems must respond to changes in frequency of the primary noise with which they are working. In other words, the primary non-stationary noise vary, hence to get an acceptable cancellation we must use some kind of adaptive system, that carried out many operations at a high speed. To satisfy the requirements those ANC systems, it should be used digital signal processors (PDSs) compute based on adaptive algorithms and adaptive filters. The ability of an adaptive filter to operate and respond satisfactorily to an unknown environment, and variations that may be involved in signal reference, to make a powerful adaptive filter for signal processing and control applications. There are various types of adaptive filters but generally all share the characteristic of working with an input signal (input vector), and a desired response (output vector), these two signals are used to compute an estimate of error (error signal), which allows to control the coefficients of the adjustable filter.

In this chapter, it's proposed a new Hybrid active noise control (ANC) system. The main feature of the proposed system is the consideration of both, the online secondary path modeling and the acoustic feedback. The objective is to realize improved performance at a reasonable computational cost in a Hybrid ANC system that considers two of the more important troubles of the ANC. In the proposed system, two least mean square (LMS) adaptive filters are used in the noise control process, one for the Feedforward stage and the other for the Feedback stage, both of them use the same error signal as used in the 
adaptation of the modeling filter (Romero et al, 2008). Moreover, the method of online secondary path modeling is an adaptation of the method proposed by (Akhtar et al, 2006) in which a variable step size (VSS-LMS) algorithm is used to adapt the modeling filter. With the VSS-LMS algorithm a small step size is required initially for the modeling filter and later its value is increased accordingly. The combination of the Feedback and Feedforward stages, gives to the system a solid robustness in frequency domain. Finally, the computer simulations are presented to show the success of the proposed system.

\section{Adaptive filters}

\subsection{What is an adaptive filter?}

An adaptive filter responds to changes in its parameters, like for example: its resonance frequency, input signal or transfer function that varies with time. This behavior is possible due to the adaptive filter coefficients vary over time and are updated automatically by an adaptive algorithm.

Therefore, these filters can be used in applications where the input signal is unknown or not necessarily stationary. An adaptive filter is made up of two parts: a digital filter and an adaptive algorithm. The block diagram of an adaptive filter is shown in Figure 1, we can see that the adaptive algorithm needs, two input signals, $x(n)$ and $e(n)$ as its references to set the parameters of the digital filter and update its coefficients.

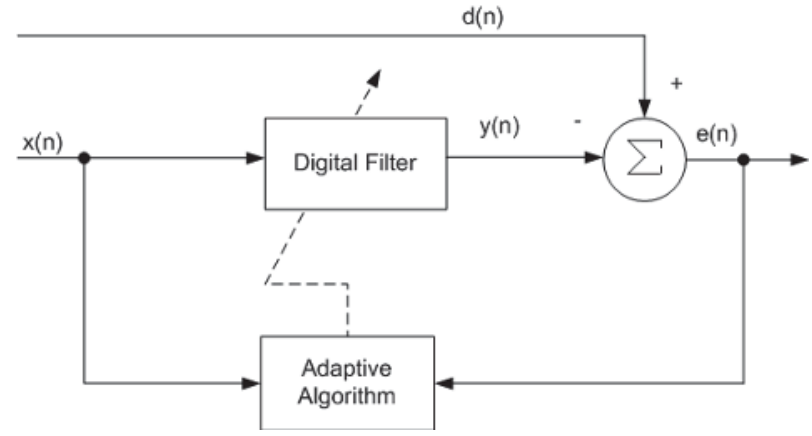

Fig. 1. Adaptive Filter

\subsection{Least Mean Square algorithm}

The Least Mean Square (LMS) algorithm is part of the stochastic gradient algorithm family that does not require the knowledge of autocorrelation function or inverse function arrays to update the coefficients values of an adaptive filter.

From Figure 1 we define that a Finite Impulse Response (FIR) adaptive filter with LMS algorithm can describes is update equation as

$$
\overline{\mathrm{w}}(n+1)=\overline{\mathrm{w}}(n)+\mu \overline{\mathrm{x}}(n) e(n)
$$

where $\overline{\mathrm{x}}(n) \equiv[x(n), x(n-1), \ldots, x(n-L+1)]^{\mathrm{T}}$, is the $L$ sampled reference signal vector, and $x(n)$ is the reference signal obtained by the reference microphone, $\mu$ is the step size of the adaptive algorithm (Kuo \& Morgan, 1996), $e(n)$ is the residual noise signal given as 


$$
e(n)=d(n)-y(n)
$$

and finally $\overline{\mathbf{w}}(n) \equiv\left[w_{0}(n), w_{1}(n), \ldots, w_{L-1}(n)\right]^{\mathrm{T}}$, is the tap-weight vector. LMS algorithm also has the advantage of being simple and requires no differences or averages from the input signal (Kuo \& Morgan, 1996).

\subsection{ANC Systems}

\subsubsection{Types of systems}

\subsubsection{Feedforward systems}

Based on the application of identification of adaptive filters, the Feedforward system described in Figure 2, exemplifies this ANC system in an acoustic media, where unwanted signal emitted by the source noise is monitored by both the reference and error microphones and processed by the adaptive filter in the block of ANC.

Figure 3 shows in a simplified form a Feedforward ANC system where the digital filter $W(z)$ is used to estimate the unknown plant $P(z)$. It is assumed that the plant and the filter have the same input signal $x(n)$.

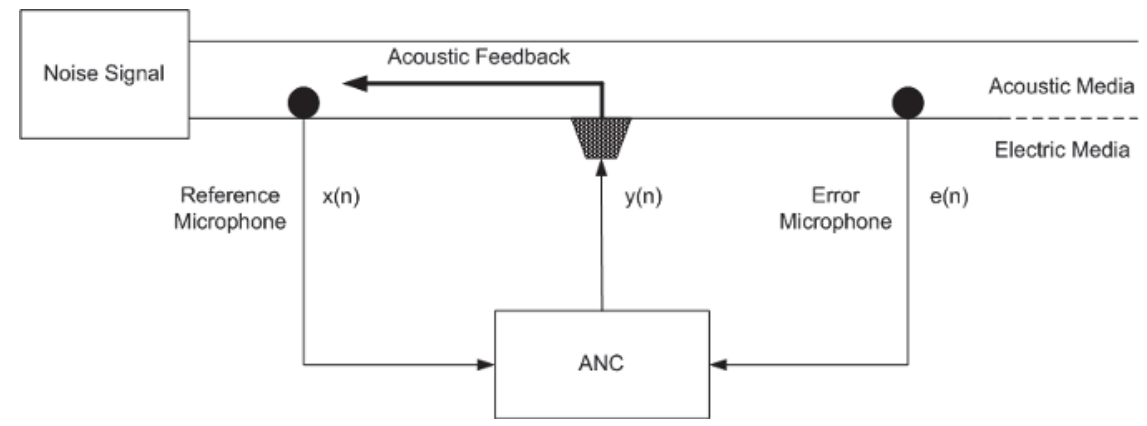

Fig. 2. Feedforward ANC Process

Moreover, we introduce the algorithm Filtered-X Least Mean Square (FXLMS), a variant of the LMS algorithm discussed in (Kuo \& Morgan, 1996); FXLMS solve the problem of secondary path described as the set of transformations experienced by the signal response of the filter and adaptive signal mistake of passing a domain electric acoustic one. In this electroacustic process, the signal can be delayed or altered, so that's why is necessary to minimize these effects. The technique of the algorithm FXLMS consists of placing a filter with the same characteristics as the secondary path in the reference signal that leads towards the adaptive filter, like is shown in Fig 3.

The filter $\hat{S}(z)$ in Figure 3, is the estimation of the secondary path defined by the filter $S(z)$. By taking this consideration, the actualization of the filter $W(z)$ becomes as:

$$
\overline{\mathrm{w}}(n+1)=\overline{\mathrm{W}}(n)+\mu \overline{\hat{\mathrm{x}}}(n) e(n)
$$

where:

$$
\overline{\hat{x}}(n)=\hat{s}(n) * \overline{\mathrm{x}}(n)
$$




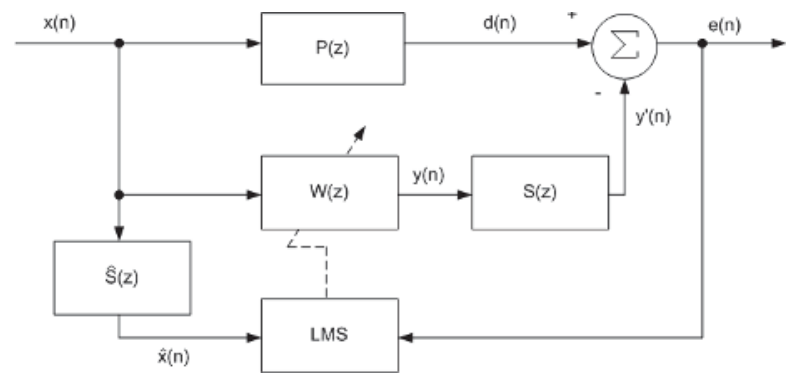

Fig. 3. Feedforward ANC System with FXLMS Algorithm

\subsubsection{Feedback systems}

There are some applications where it is not possible to take into account the reference signal from the primary source of noise in a Feedforward ANC system, perhaps because it is difficult to access to the source, or there are several sources that make it difficult to identify a specific one by the reference microphone. One solution to this problem is the one that introduced a system that predicts the input signal behavior, this system is know has the Feedback ANC system which is characterized by using only one error sensor and a secondary source (speaker) to achieve the noise control process.

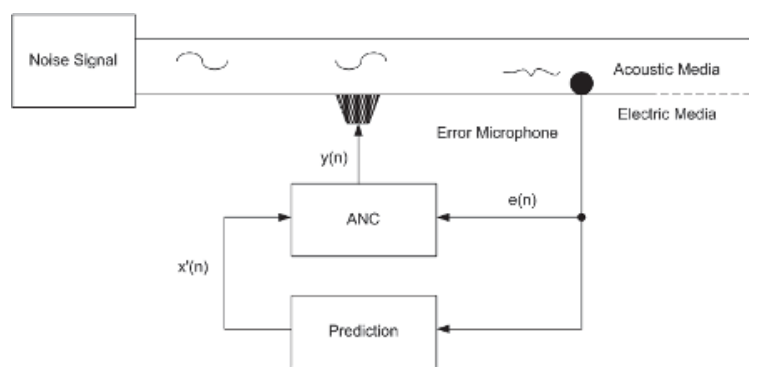

Fig. 4. Feedback ANC Process

Figure 5 describes a Feedback ANC system with FXLMS algorithm, in which $d(n)$ is the noise signal, $e(n)$ is the error signal defined as the difference between $d(n)$ and the $y^{\prime}(n)$,

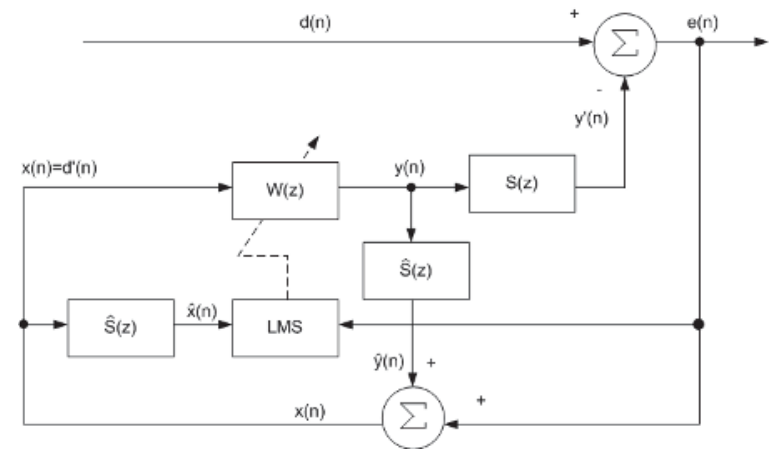

Fig. 5. Feedback ANC System with FXLMS Algorithm 
the output signal of the adaptive filter once it already has crossed the secondary path. Finally, the input signal of the adaptive filter is generated by the addition of the error signal and the signal resulting from the convolution between the secondary path $\hat{S}(z)$ and the estimated output of adaptive filter $y(n)$.

\subsubsection{Hybrid system}

A Hybrid system consists of one identification stage (Feedforward) and one prediction (Feedback) stage. This combination of both Feedback and Feedforward systems needs two reference sensors: one related to the primary source of noise and another with the residual error signal.

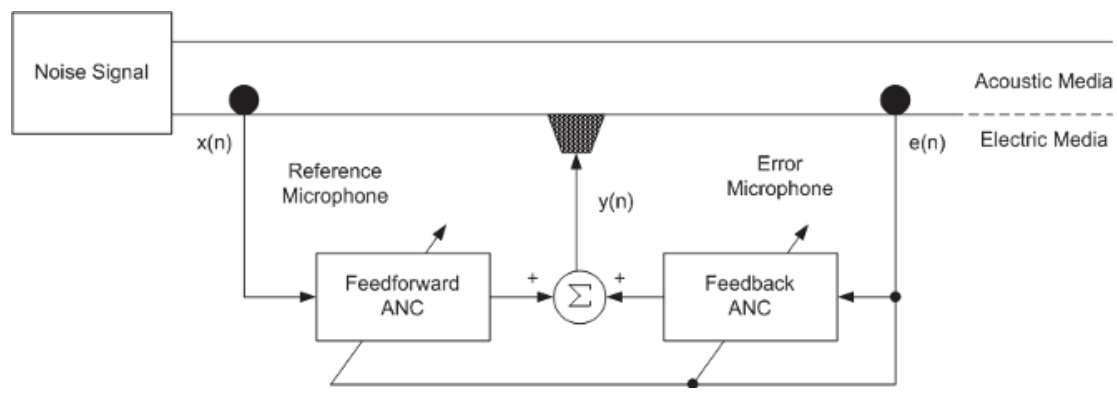

Fig. 6. Hybrid ANC Process

Figure 7 shows the detailed block diagram of an ANC Hybrid System in which it is possible to observe the basic systems (Feedforward, Feedback) involved in this design. The attenuation signal resulting from the addition of the two outputs $W(z)$ and $M(z)$ of adaptive filters is denoted by $y(n)$. The filter $M(z)$ represents the adaptive filter Feedback process, while the filter $W(z)$ represents the Feedforward process. The secondary path consideration in the basic ANC systems is also studied in the design of the Hybrid system and is represented by the transfer function $S(z)$.

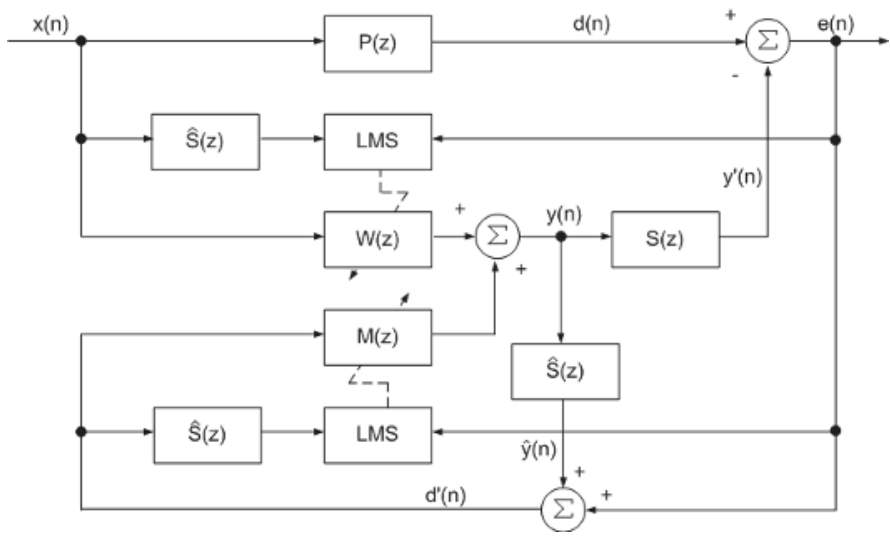

Fig. 7. Hybrid ANC System with FXLMS Algorithm 
As we can see, the block diagram of the Hybrid ANC system from Figure 8 also employs the FXLMS algorithm to compensate the possible delays or troubles that the secondary path provokes.

\subsubsection{ANC problematic}

\subsubsection{Acoustic feedback}

This characteristic is present in an ANC Feedforward system; Figure 2 shows that the contribution of the attenuation signal $y(n)$, causes a degradation of the system response because this signal is present in the microphone reference. Two possible solutions to this problem are: the neutralization of acoustic feedback and the proposal for a Hybrid system that by itself has a better performance in the frequency range of work and the level of attenuation. To solve this issue we analyze a Hybrid system like shown in the Figure 8, where $F(z)$ represents the transfer function of the Feedback process.

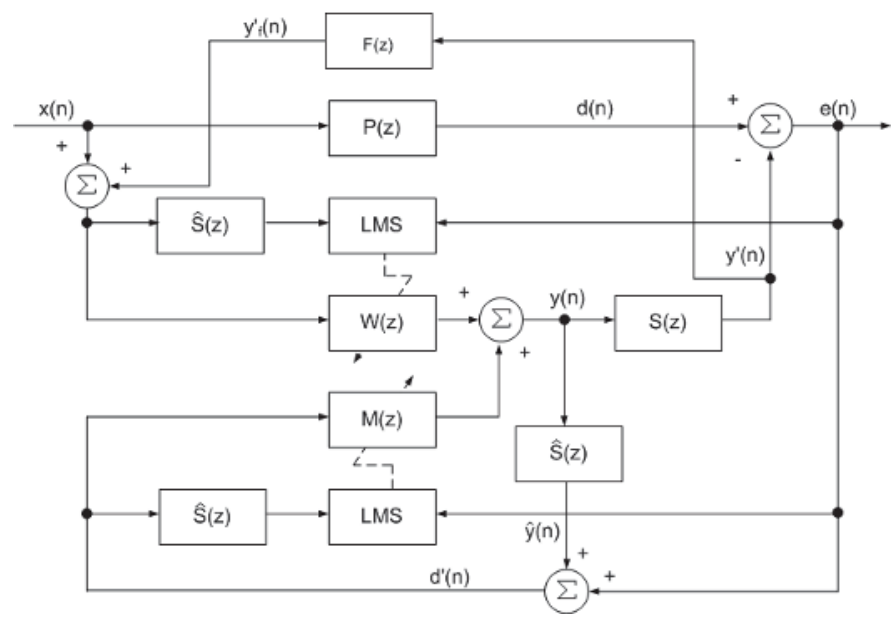

Fig. 8. Hybrid ANC System with Acoustic Feedback

\subsubsection{Secondary path modeling}

As previously mentioned, the process that makes the signal resulting from the adaptive filter $y(n)$ into $e(n)$, is defined as a secondary path. This feature takes in consideration, digital to analog converter, reconstruction filter, the loudspeaker, amplifier, the trajectory of acoustic loudspeaker to the sensor error, the error microphone, and analog to digital converter. There are two techniques for estimating the secondary path, both techniques have their tracks that offer more comprehensive and sophisticated methods in certain aspects, these techniques are: offline secondary path modeling and the online secondary path modeling. The first one is done by a Feedforward system where the plant now is $S(z)$ and the coefficients of the adaptive filter are the estimation of the secondary path, like shown in Figure 9: 


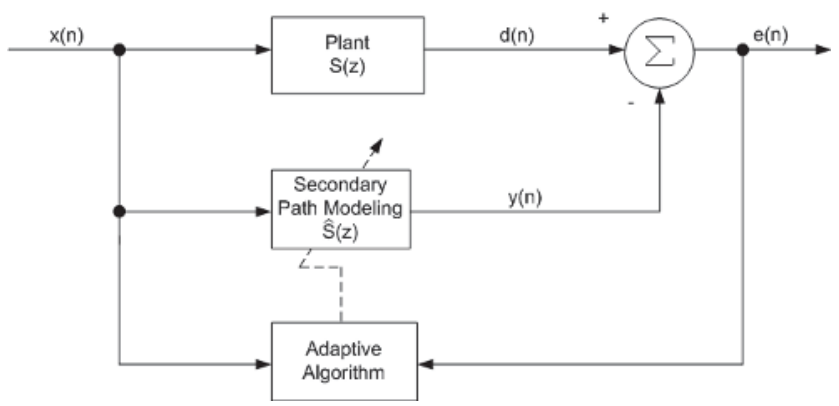

Fig. 9. Offline Secondary Path Modeling

For online secondary path modeling we study two methods: Eriksson's method (Eriksson et al, 1988) and Akhtar's method (Akthar et al, 2006). Figure 10 shows the Eriksson's Method where first a zero mean white noise $v(n)$, which is not correlated with the primary noise is injected at the entrance to the secondary loudspeaker. Secondly, $x(n)$ represents the discrete output form reference microphone, also known as reference signal; $\overline{\mathrm{p}}(n)=[p(n), p(n-1), \ldots, p(n-L-N+1)]^{\mathrm{T}}$ is the vector containing the impulse response of the primary path from the digital output microphone reference to the exit of the microphone error. The vector composed of the impulse response of the secondary path of the digital output of the loudspeaker secondary to the exit discrete microphone error is defined as $\bar{s}(n)=[s(n), s(n-1), \ldots, s(n-L-N+1)]^{\mathrm{T}}$. Moreover, the adaptive filter $\overline{\mathrm{w}}(n)$ is in charge of the noise control process, and it is defined as $\overline{\mathrm{w}}(n) \equiv[w(0), w(1), \ldots, w(L-1)]^{\mathrm{T}}$ where $L$ represents the length of the filter. The signal $d(n)$ is output $p(n)$ due to $x(n)$; the signal that cancels, $y(n)$, is output of the noise control process due $x(n)$. It is important to consider the update of the coefficients of the secondary path filter defined as:

$$
\overline{\hat{\mathrm{s}}}(n+1)=\overline{\hat{\mathrm{s}}}(n)+\mu_{\mathrm{s}} \overline{\mathrm{v}}(n)\left[v^{\prime}(n)-\hat{v}(n)\right]+\mu_{\mathrm{s}} \overline{\mathrm{v}}(n) \eta(n)
$$

where $v^{\prime}(n)=v(n) * s(n)$ and $\hat{v}^{\prime}(n)=v(n) * \hat{s}(n) ; *$ denotes convolution.

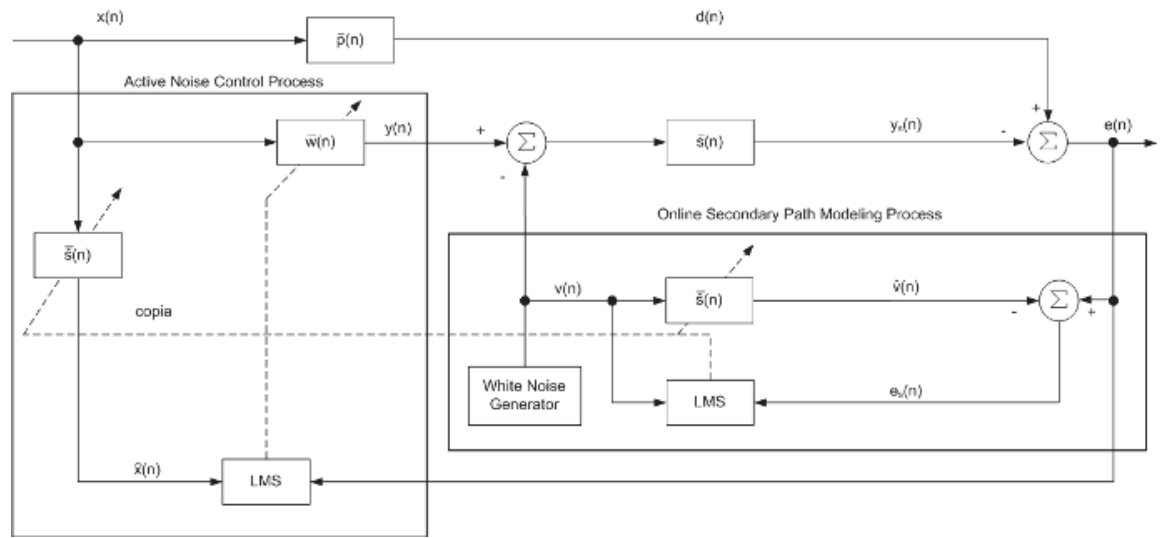

Fig. 10. ANC System with Online Secondary Path Modeling (Eriksson's Method) 
For the Akhtar's method the noise control adaptive filter is updated using the same error signal that the adaptive filter that estimated the secondary path. At the same time, an algorithm LMS variable sized step (VSS-LMS) is used to adjust the filter estimation of the secondary path. The main reason for using an algorithm VSS-LMS responds to the fact that the distorted signal present at the desired filter response of the secondary path decreases in nature, ideally converge to zero. Ec. 6 describes the coefficients vector of the noise control filter as:

$$
\begin{aligned}
\overline{\mathrm{w}}(n+1) & =\overline{\mathrm{w}}(n)+\mu_{w}\left[d(n) \overline{\hat{\mathrm{x}}}(n)-y^{\prime}(n) \overline{\hat{\mathrm{x}}}(n)\right] \\
& +\mu_{w}\left[v^{\prime}(n)-\hat{v}(n)\right]
\end{aligned}
$$

Is important to realize that the contribution of the white noise, $v^{\prime}(n)$ and $\hat{v}(n)$ is uncorrelated with the input signal $x(n)$, so the Akhtar's method reduces this perturbation in the coefficients vector of the filter $W(z)$ when the process of secondary path modeling is such that $\hat{S}(z) \approx S(z)$, in this moment, $v^{\prime}(n)-\hat{v}(n) \approx 0$ and the noise control process is completely correlated.

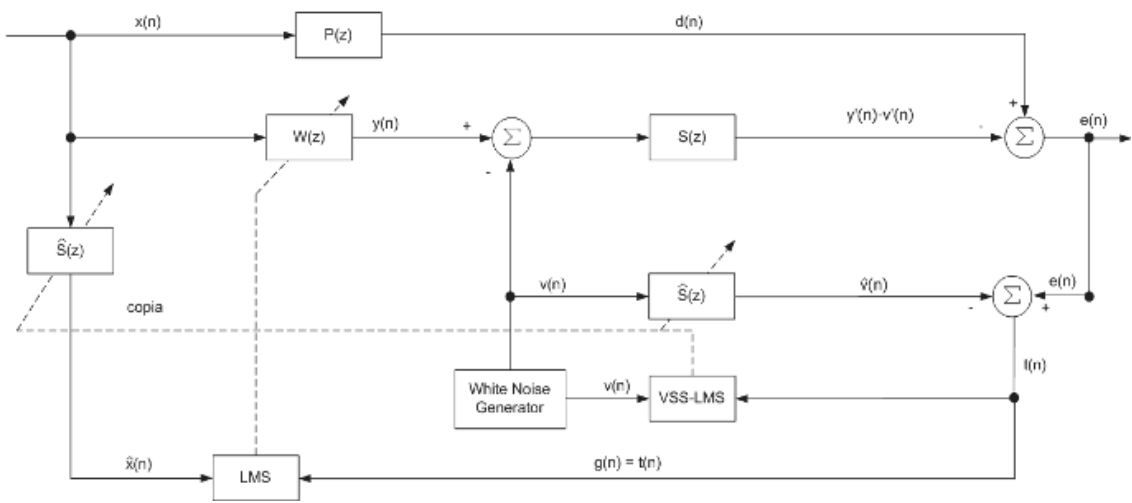

Fig. 11. ANC System with Online Secondary Path Modeling (Akhtar's Method)

\subsubsection{Proposed Hybrid system}

As a result of both considerations, the acoustic feedback and the online secondary path modeling, here we suggest a Hybrid ANC system with online secondary path modeling and acoustic feedback. The idea is to conceive a new robust system like the block diagram of the Figure 12 shows.

Its possible to observe from Figure 12 that the same signal, $a(n)$, is used as the error signal of the adaptive filter $W(z)$ which intervenes in the identification stage of the Feedforward system present in the proposed configuration. Also it's important to realize that in our design we have three FIR adaptive filters $W(z), M(z)$ and $\hat{S}(z)$. The first one intervenes in the Feedforward process, $M(z)$ is part of the Feedback process; $\hat{S}(z)$ represents the online secondary path modeling adaptive filter. Finally the block $F(z)$ is the consideration of the acoustic feedback. 


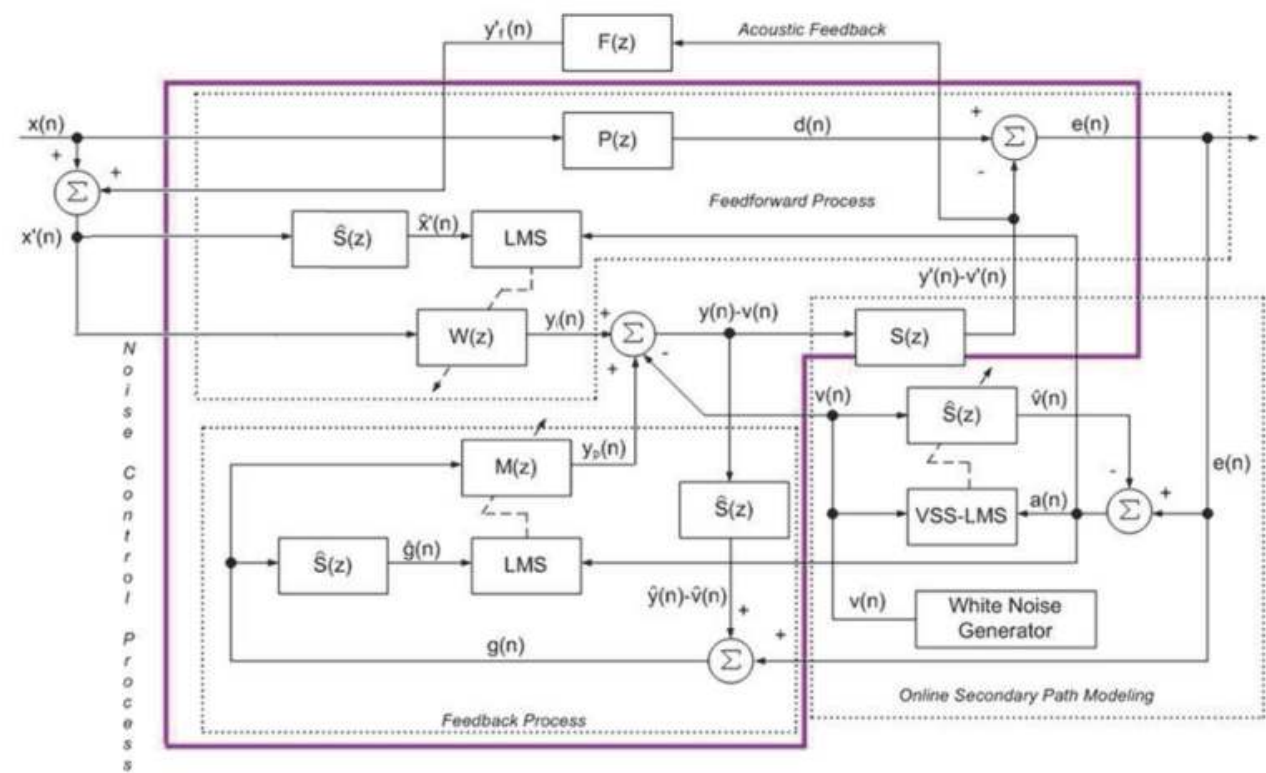

Fig. 12. A Hybrid Active Noise Control System with Online Secondary Path Modeling and Acoustic Feedback (Proposed System)

On the basis of the Figure 12, we can see that the error signal of all the ANC system is defined as:

$$
e(n)=d(n)+[v(n)-y(n)] * s(n)
$$

where $d(n)$ is the desired response, $v(n)$ is the white noise signal, $s(n)$ is the finite impulse response of the secondary path filter $S(z)$ and $y(n)$ is the resultant signal of the acoustic noise control process that achieves attenuate the primary noise signal and is defined as:

$$
y(n)=y_{i}(n)+y_{p}(n)
$$

where $y_{i}(n)=\overline{\mathrm{W}}^{\mathrm{T}}(n) \overline{\mathrm{x}}^{\prime}(n)$ represents the signal resultant of the Feedforward process, once again $\overline{\mathbf{w}}(n) \equiv\left[w_{0}(n), w_{1}(n), \ldots, w_{L-1}(n)\right]^{\mathrm{T}}, \quad$ is the tap-weight vector, $\bar{x}^{\prime}(n) \equiv\left[x^{\prime}(n), x^{\prime}(n-1), \ldots, x^{\prime}(n-L+1)\right]^{\mathrm{T}}$ is the $L$ sample reference signal vector of the Feedforward stage and $x^{\prime}(n)=x(n)+y_{f}^{\prime}(n)-v^{\prime}{ }_{f}(n)$ is the reference signal that already considers the effects of the acoustic feedback. By the way, as a result of the acoustic feedback consideration we expressed:

$$
\begin{aligned}
& y_{f}^{\prime}(n)=y^{\prime}(n) * f(n) \\
& v_{f}^{\prime}(n)=v^{\prime}(n) * f(n)
\end{aligned}
$$


Both Ec. 9 and Ec. 10 contain $f(n)$, the finite impulse response of the acoustic feedback filter; moreover $y^{\prime}(n)$ and $v^{\prime}(n)$ are the signals that already have cross $S(z)$, the secondary path filter.

In the other and, for the Feedback stage we have that $y_{p}(n)=\overline{\mathrm{m}}^{\mathrm{T}}(n) \overline{\mathrm{g}}(n)$ is the noise control signal for this process, where $\overline{\mathrm{m}}(n) \equiv\left[m_{0}(n), m_{1}(n), \ldots, m_{M-1}(n)\right]^{\mathrm{T}}$ is the tap-weight vector of length $M$ of the filter $M(z) ; \bar{g}(n) \equiv[g(n), g(n-1), \ldots, g(n-M+1)]^{\mathrm{T}}$ is the sample reference signal for this adaptive filter and $g(n)=e(n)+\hat{y}(n)-\hat{v}(n)$ is the reference signal, where:

$$
\begin{aligned}
& \hat{v}(n)=v(n) * \hat{s}(n) \\
& \hat{y}(n)=y(n) * \hat{s}(n)
\end{aligned}
$$

Once again as a result of the FXLMS algorithm, the Ec. 11 and Ec. 12 consider the signals $y(n)$ and $v(n)$ once both already have cross the estimation of the secondary path defined by $\hat{S}(z)$.

The advantages of using the Akhtar's method (Akthar et al, 2006 and Akthar et al, 2004), for the secondary path modeling in our proposed system are reflected in the VSS-LMS algorithm that allows the modeling process to selects initially a small step size, $\mu_{s}(n)$, and increases it to a maximum value in accordance with the decrease in $\left[d(n)-y^{\prime}(n)\right]$. If the filter $W(z)$ is slow in reducing $\left[d(n)-y^{\prime}(n)\right]$, then step size may stay to small value for more time. Furthermore, the signal $a(n)=e(n)-\hat{v}(n)$ is the same error signal for all the adaptive filters involved in our system, $W(z), M(z)$ and $\hat{S}(z)$, the reason to use this signal is that for $W(z),\left[v^{\prime}(n)-v(n)\right]<v^{\prime}(n)$ compared with the Eriksson's method, so when $\hat{S}(z)$ converges as $\hat{S}(z) \approx S(z)$, ideally $v^{\prime}(n) \approx v(n) \Rightarrow v^{\prime}(n)-v(n) \rightarrow 0$. The bottom equations describe the update vector equations for the three adaptive filters:

$$
\begin{aligned}
\overline{\mathrm{w}}(n+1)= & \overline{\mathrm{w}}(n)+\mu_{w} \overline{\hat{\mathrm{x}}}(n)\left[d(n)-y^{\prime}(n)\right] \\
& +\mu_{w} \overline{\hat{\mathrm{x}}}(n)\left[v^{\prime}(n)-\hat{v}(n)\right] \\
\overline{\mathrm{m}}(n+1)= & \overline{\mathrm{m}}(n)+\mu_{m} \overline{\hat{\mathrm{g}}}(n)\left[d(n)-y^{\prime}(n)\right] \\
& +\mu_{m} \overline{\hat{\mathrm{g}}}(n)\left[v^{\prime}(n)-\hat{v}(n)\right] \\
\overline{\hat{\mathrm{s}}}(n+1)= & \overline{\hat{\mathrm{s}}}(n)+\mu_{s} \overline{\mathrm{v}}(n)\left[v^{\prime}(n)-\hat{v}(n)\right] \\
& +\mu_{s} \overline{\mathrm{v}}(n)\left[d(n)-y^{\prime}(n)\right]
\end{aligned}
$$

Although the Ec. 13 shows that when $\hat{S}(z)$ converges the whole control noise process of the system is not perturbed by the estimation process of $\hat{S}(z)$, it is significant to identify that the online secondary path modeling is degraded by the perturbation of $\eta(n)=\mu_{s} \overline{\mathrm{v}}(n)\left[d(n)-y^{\prime}(n)\right]$.

\section{Performance indicators}

\subsection{Classical analysis}

This section presents the simulation experiments performed to verify the proposed method. The modeling error was defined by Akhtar (Akthar et al, 2006), as: 


$$
\Delta S(d B)=10 \log _{10}\left[\frac{\sum_{i=0}^{M-1}\left[s_{i}(n)-\hat{s}_{i}(n)\right]^{2}}{\sum_{i=0}^{M-1}\left[s_{i}(n)\right]^{2}}\right]
$$

First, an offline modeling was used to obtain FIR representations of tap weight length 20 for $P(z)$ and of tap weight length 20 for $S(z)$. The control filter $W(z)$ and the modeling filter $\hat{S}(z)$ are FIR filters of tap weight length of $L=20$ both of them. A null vector initializes the control filter $W(z)$. To initializes $\hat{S}(z)$, offline secondary path modeling is performed which is stopped when the modeling error has been reduced to $-5 \mathrm{~dB}$. The step size parameters are adjusted by trial and error for fast and stable convergence.

\begin{tabular}{|c|c|c|}
\hline Case & $\begin{array}{c}\text { Step Size: } \\
\mu_{w}, \mu_{m}\end{array}$ & $\begin{array}{c}\text { Step Size: } \\
\mu_{s}\end{array}$ \\
\hline Case 1 & 0.01 & $(0.01-0.10)$ \\
\hline Case 2 & 0.01 & $(0.01-0.15)$ \\
\hline Case 3 & 0.01 & $(0.01-0.20)$ \\
\hline
\end{tabular}

Table 1. Filters Step Size Used in Classical Analysis

\subsection{Proposed analysis}

It is important to mention that the system is considered within the limitations of a duct, or one-dimensional waveguide, whose limitations are relatively easy to satisfy, as the distance between the control system and the primary sources is not very important. A duct is the simplest system, since it only involves one anti-noise source and one error sensor. (Kuo \& Morgan, 1999). The amount of noise reduction will depend on the physical arrays of the control sources and the error sensors. Moving their positions affects the maximum possible level of noise reduction and the system's stability (the rate at which the controller adapts to system changes).

In order to decide which control system is the best, the properties of the noise to be cancelled must be known. According to (Kuo \& Morgan, 1999), it is easier to control periodic noise; practical control of random or transitory noise is restricted to applications where sound is confined, which is the case of a duct.

The noise signals used for the purposes of this work are sorted into one of three types, explained next. This classification is used by several authors, amongst whom are (Kuo \& Morgan, 1999) and (Romero et al, 2005), as well as companies such as (Brüel \& Kjaer Sound \& Vibration Measurement, 2008).

1. Continuous or constant: Noise whose sound pressure level remains constant or has very small fluctuations along time.

2. Intermittent or fluctuant: Noise whose level of sound pressure fluctuates along time. These fluctuations may be periodic or random.

3. Impulsive: Noise whose level of sound pressure is presented by impulses. It is characterized by a sudden rise of noise and a brief duration of the impulse, relatively compared to the time that passes between impulses.

Various articles on the subject of ANC were taken into consideration before establishing three main analysis parameters to determine the hybrid system's performance:

a. Nature of the test signals; as far as the test signals are concerned, the system was tested with several real sound signals taken from an Internet database (Free sounds effects \& 
music, 2008). The sound files were selected taking into consideration that the system is to be implemented in a duct-like environment.

b. Filter order; it is important to evaluate the system under filters of different orders. In this case, 20 and 32 coefficients were selected, which are low numbers given the fact that the distance between the noise source and the control system is not supposed to be very large. For 20th order filters, two cases were considered.

c. Nature of the filter coefficients; on a first stage, the coefficients were normalized; this means that they were set randomly with values from -1 to 1 . Next, the coefficients were changed to real values taken from a previous study made on a specific air duct (Kuo \& Morgan, 1996).

Thus, the tests were carried out on three different stages:

1. Analysis with real signals and filters with 20 random coefficients;

2. Analysis with real signals and filters with 32 random coefficients; and

3. Analysis with real signals and filters with 20 real coefficients.

The simulation results are presented according to the following parameters:

1. Mean Square Error (MSE); and

2. Modeling error from online secondary path modeling.

Equation 17 shows the MSE calculation, given by the ratio between the power of the error signal, and the power of the reference signal.

$$
\operatorname{MSE}(d B)=10 \log _{10}\left[\frac{\sum_{i=0}^{M-1}\left[e_{i}(n)\right]^{2}}{\sum_{i=0}^{M-1}\left[x_{i}(n)\right]^{2}}\right]
$$

Equation 18 is the calculation for the Modeling error, given by the ratio of the difference between the secondary path and its estimation, and the secondary path as defined by Akthar (Akthar et al, 2006):

$$
\Delta S(d B)=10 \log _{10}\left[\frac{\sum_{i=0}^{M-1}\left[s_{i}(n)-\hat{s}_{i}(n)\right]^{2}}{\sum_{i=0}^{M-1}\left[s_{i}(n)\right]^{2}}\right]
$$

\section{Analysis of results}

\subsection{Classical references}

In this cases, according bibliography, three sceneries are explained.

\subsubsection{Case 1}

Here the reference signal is a senoidal signal of $200 \mathrm{~Hz}$. A zero mean uniform white noise is added with SNR of $20 \mathrm{~dB}$, and a zero mean uniform white noise of variance 0.005 is used in the modeling process. Figure 13a shows the curves for relative modeling error $\Delta S$, the corresponding curves for the cancellation process is shows in Figure 13b. In iteration 1000 it is performed a change on the secondary path. 


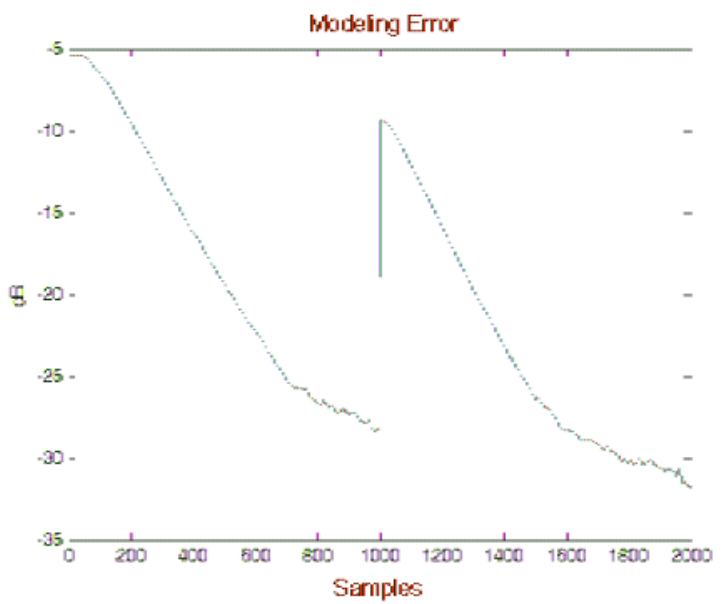

Fig. 13.a Relative Modeling Error

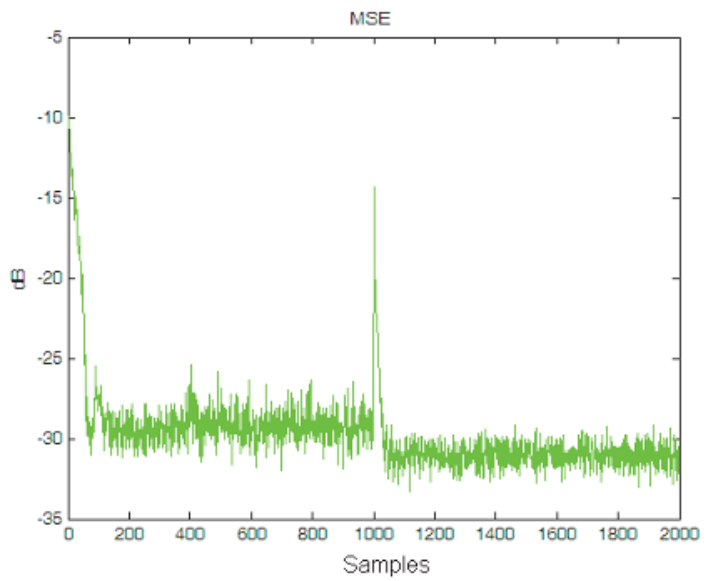

Fig. 13.b Attenuation Level

\subsubsection{Case 2}

In this case the reference signal is a narrow band sinusoidal signal with frequencies of 100,200 , $400,600 \mathrm{~Hz}$. A zero mean uniform white noise is added with SNR of 20dB, and a zero mean uniform white noise of variance 0.005 is used in the modeling process. The simulations results are shown in Figure 14a. In iteration 1000 it is performed a change con the secondary path. 


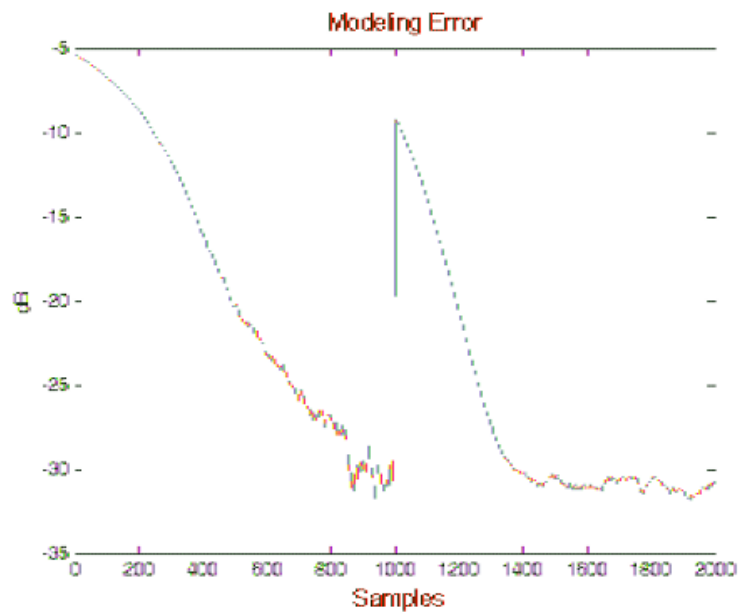

Fig. 14.a Relative Modeling Error

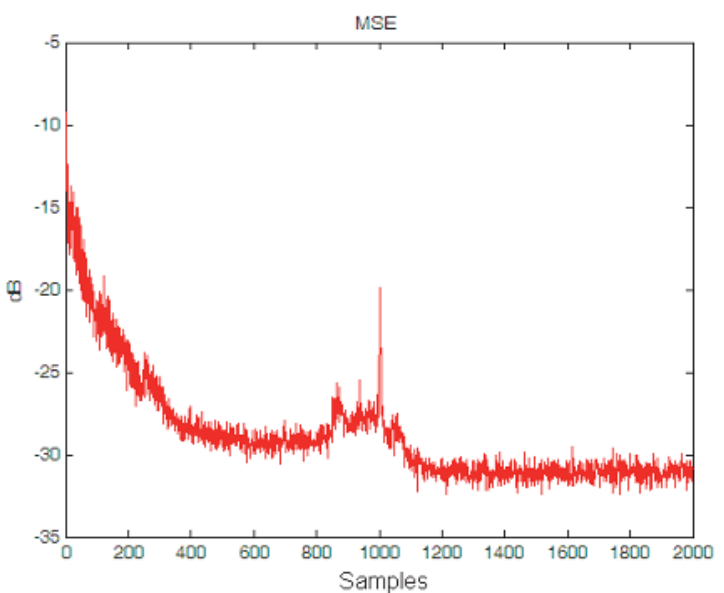

Fig. 14.b Attenuation Level

\subsubsection{Case 3}

Here we consider a motor signal for the reference signal. A zero mean uniform white noise of variance 0.005 is used in the modeling process. The simulations results are shown in Figure 15a. In iteration 1000 it is performed a change on the secondary path. 


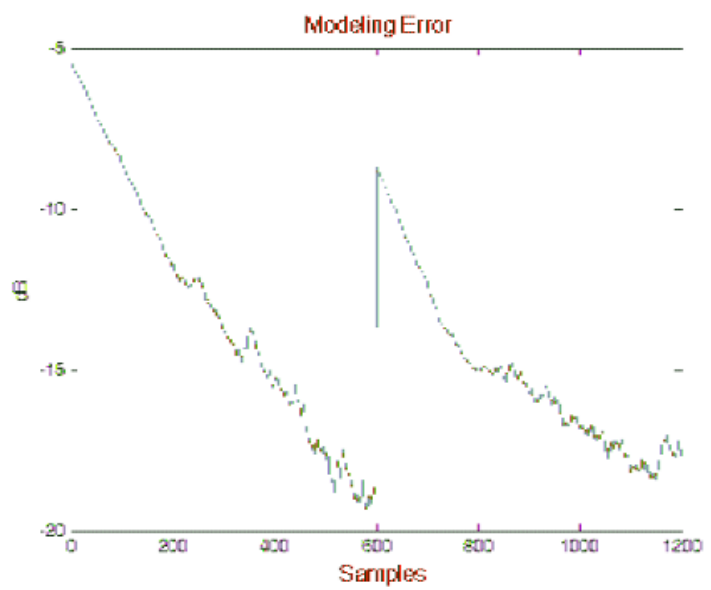

Fig. 15.a Relative Modeling Error

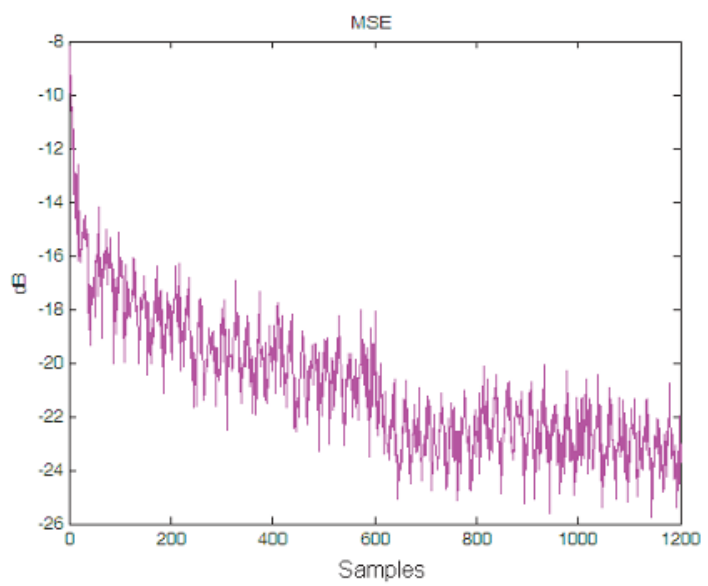

Fig. 15.b Attenuation Level

\subsection{Proposed evaluation set}

\subsubsection{Test signal characterization}

In order to characterize the hybrid system, several simulation tests were made with different real signals of each type described before. One signal of each type was selected to show the simulation results in this in this work. These three signals are the most representative case for each noise type. 
First, each signal characterization will be shown, obtained through a program written in the simulation environment Matlab®. The graphs shown for each signal are: 1) Amplitude vs. Number of samples; 2) Amplitude vs. Frequency; and 3) Power vs. Frequency. Figure 16 shows the continuous signal, which corresponds to the audio of a vacuum cleaner in use. This signal has mainly low frequency components, and the power distribution is also found within low frequencies.

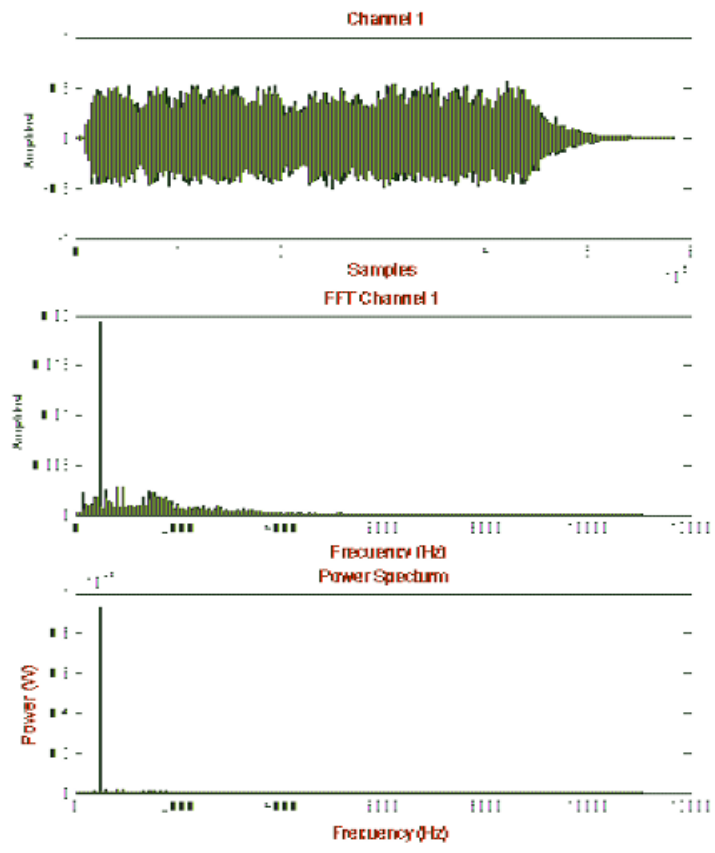

Fig. 16. Continuous Test Signal

Figure 17 shows the intermittent signal, which is the audio of a hand blender in use. This signal has relatively periodic fluctuations of different lengths. It could be considered a broadband signal because of the distribution of its frequency components, and its power is concentrated in low frequencies.

Finally, figure 18 presents the impulsive signal, given by the recording of some metallic objects falling down (a "crash" sound). There is an especially abrupt impulse by the end of the signal, which has mainly low frequency components and whose power is concentrated on low frequencies as well. 


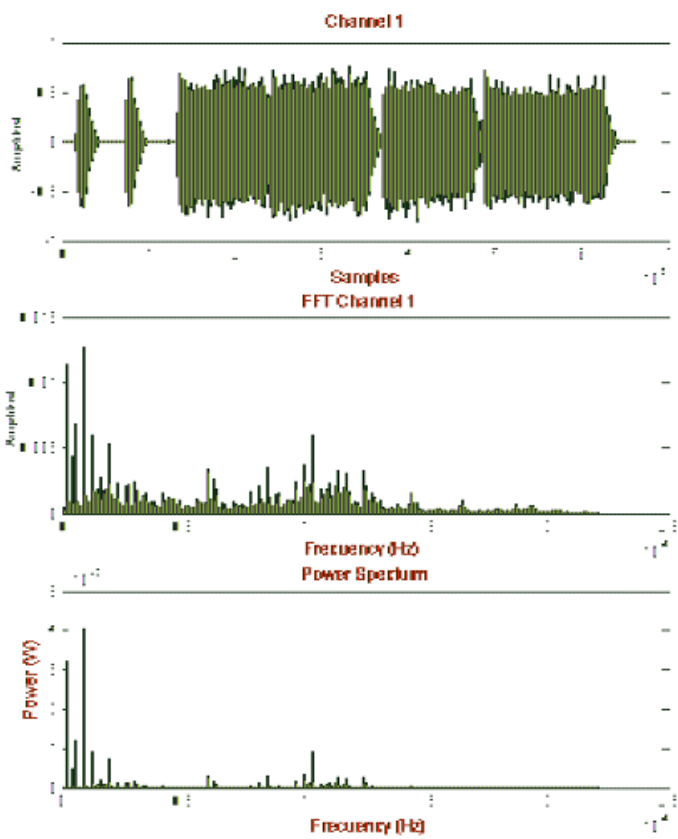

Fig. 17. Intermittent Test Signal
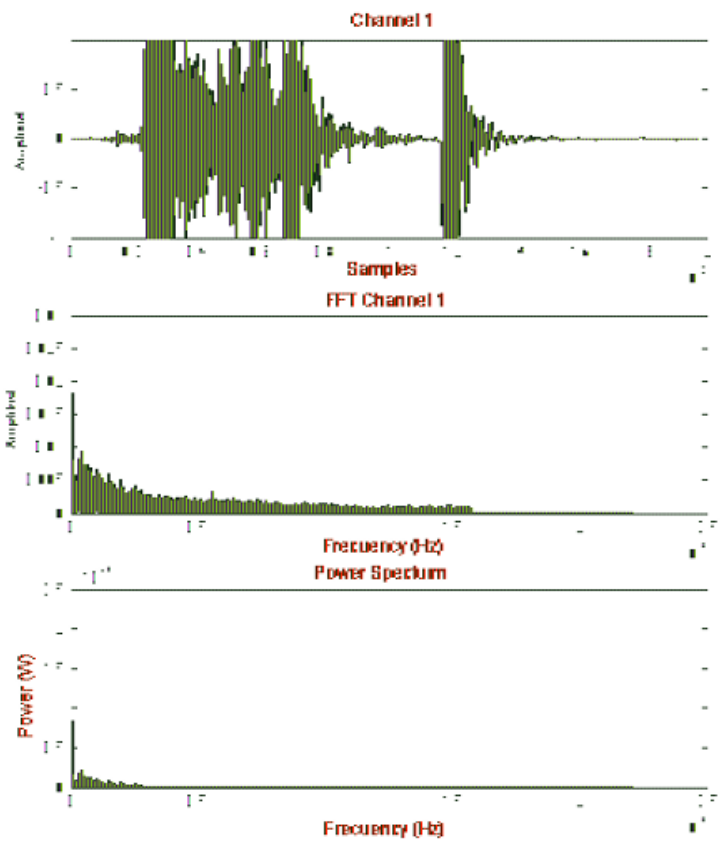

Fig. 18. Impulsive Test Signal 


\subsubsection{Filters with 20 random coefficients}

The first tests were for 20th order filters with random coefficients. The optimum values of the step sizes $\mu_{\mathrm{w}}$ and $\mu_{\mathrm{m}}$, belonging to the feedforward and feedback sections respectively, were established by trial and error. Table 2 shows the values used for each section's step size, as well as the range of values used for the step size in the secondary path filter (LopezCaudana et al, 2008).

\begin{tabular}{|c|c|c|}
\hline Signal & $\begin{array}{c}\text { Step size } \\
\mu_{\mathrm{w}}, \mu_{\mathrm{m}}\end{array}$ & $\begin{array}{c}\text { Step size } \\
\mu_{\mathrm{s}}\end{array}$ \\
\hline Continuous & 0.001 & $0.01-0.15$ \\
\hline Intermittent & 0.001 & $0.01-0.15$ \\
\hline Impulsive & 0.0001 & $0.01-0.15$ \\
\hline
\end{tabular}

Table 2. Filters Step Size Used in Proposed Analysis

On each case, a white noise with mean zero and variance equal to 0.005 was used. Also, an abrupt change in secondary path was implemented on iteration 1000 out of 2000, to test the response of the system to such changes.

The signal that gave the best response was the continuous signal. Figure 19 shows the Modeling error, while Figure 20 shows the MSE for this case. These Figures show that the system successfully achieved stability and cancelled part of the input noise signal.

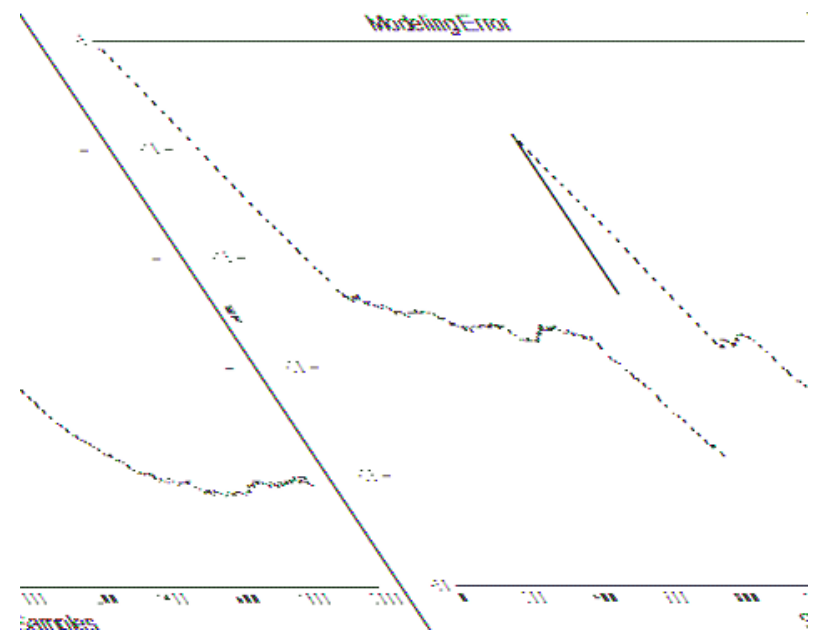

Fig. 19. Relative Modeling Error for Continuous Signal - Filters with 20 Random Coefficients 


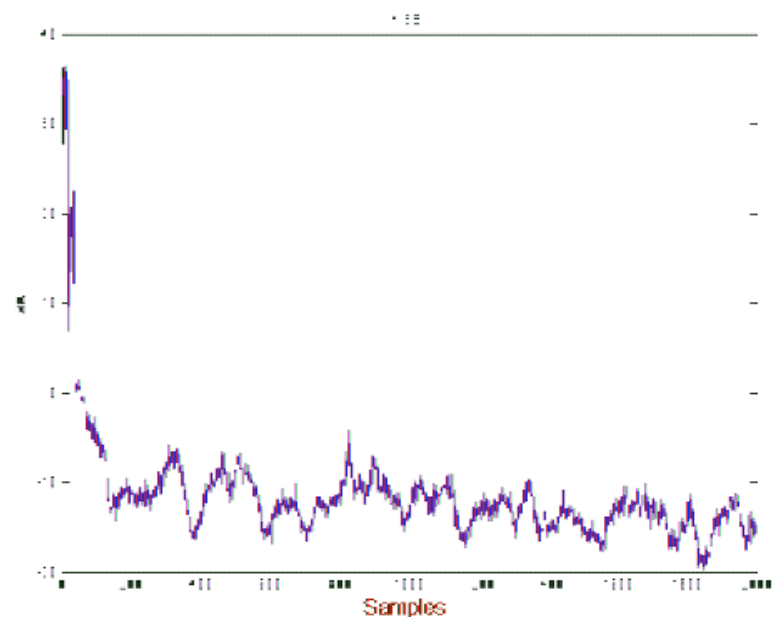

Fig. 20. MSE for Continuous Signal - Filters with 20 Random Coefficients

The response for the intermittent signal also achieved stability, despite the peaks that the signal presented at some samples, and managed to cancel part of the input noise signal as well. Figure 21 shows the Modeling error for the intermittent signal, while Figure 22 shows the MSE.

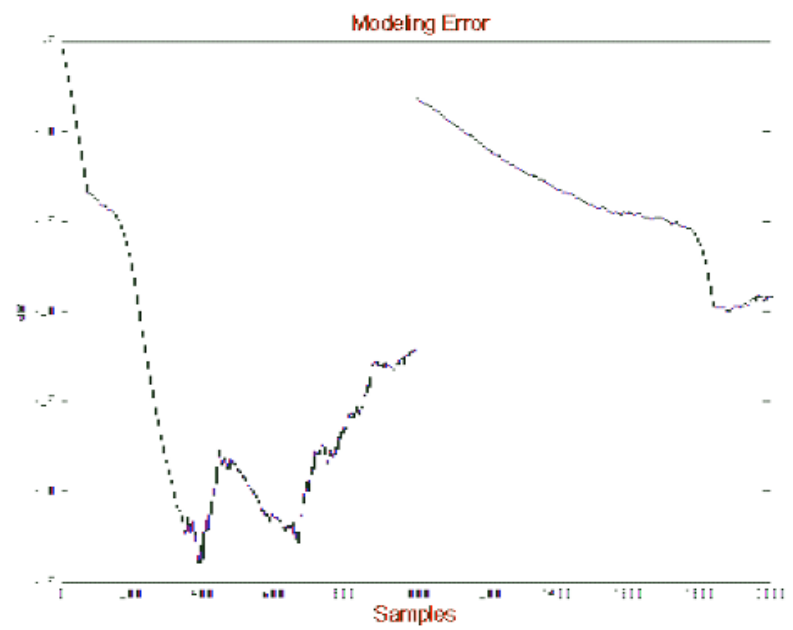

Fig. 21. Relative Modeling Error for Intermittent Signal - Filters with 20 Random Coefficients. 


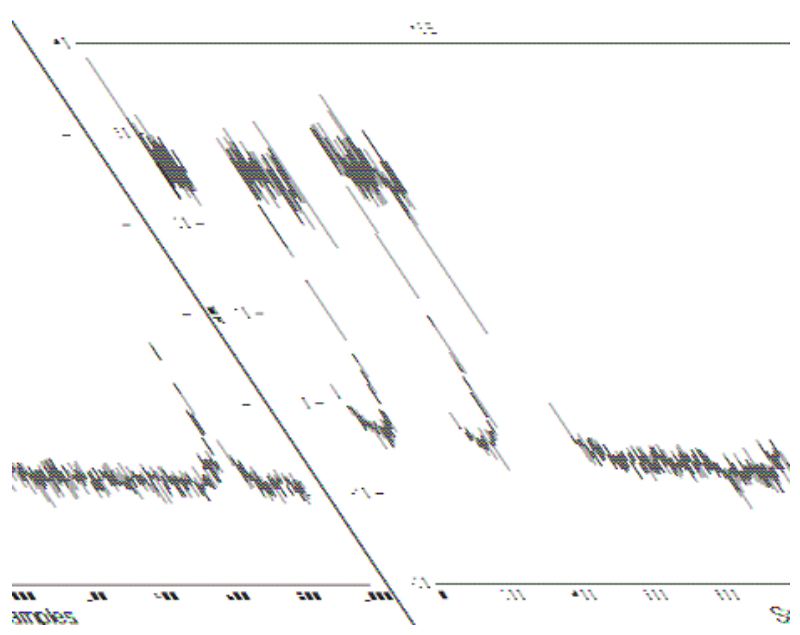

Fig. 22. MSE for Intermittent Signal - Filters with 20 Random Coefficients

However, the system presented more trouble stabilizing after the most abrupt impulse on the impulsive signal, and although it started converging, it could not cancel noise past that significant change.

\subsubsection{Filters with $\mathbf{3 2}$ random coefficients}

The next step in our set of tests was to increase the order of the filters, which means the system is taking into consideration a larger number of the duct's properties. Once again, the values of the coefficients are random from -1 to 1 .

Table 3 shows the values used for the feedforward and feedback step sizes, as well as the range of step sizes for the secondary path filter. This values were established parting from the previous test's values and, if necessary, were adjusted by trial and error.

\begin{tabular}{|c|c|c|}
\hline Signal & $\begin{array}{c}\text { Step size } \\
\mu_{\mathrm{w}}, \mu_{\mathrm{m}}\end{array}$ & $\begin{array}{c}\text { Step size } \\
\mu_{\mathrm{s}}\end{array}$ \\
\hline Continuous & 0.0001 & $0.001-0.05$ \\
\hline Intermittent & 0.001 & $0.01-0.15$ \\
\hline Impulsive & 0.00005 & $0.0001-0.05$ \\
\hline
\end{tabular}

Table 3. Filters Step Size Used in Proposed Analysis

Once again, a white noise with zero mean and variance equal to 0.005 was used for the three cases. An abrupt change in secondary path was done on iteration 1000 out of 2000.

Figure 23 shows the Modeling error response for the continuous signal, whereas Figure 24 shows the MSE for the same case. 


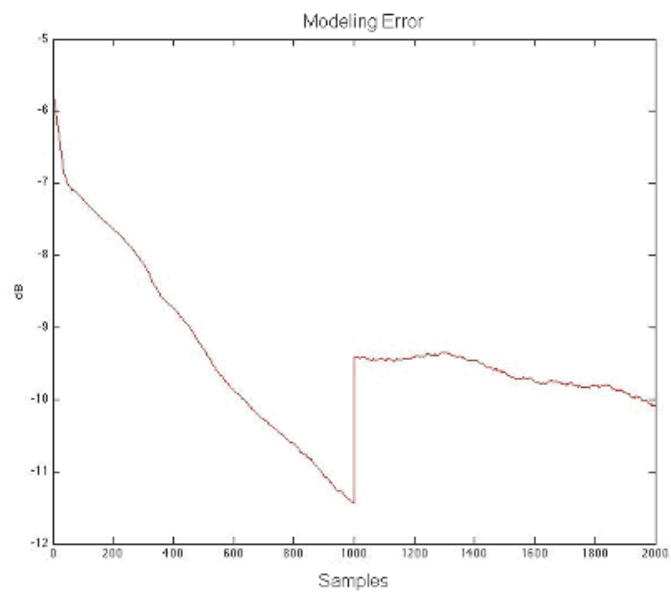

Fig. 23. Relative Modeling Error for Continuous Signal - Filters with 32 Random Coefficients

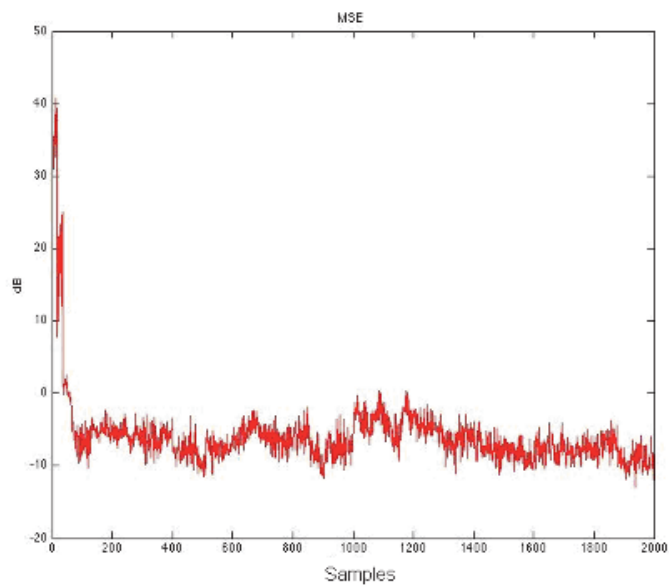

Fig. 24. MSE for Continuous Signal - Filters with 32 Random Coefficients

It can be observed that the system was, again, able to achieve stability as well as cancel noise. The step size was reduced by an order of ten in this case, probably due to the fact that it is a more accurate analysis than the previous test, because of the larger filter order. In the case of the intermittent input signal, the step size values did not need to be altered, and the 
hybrid system achieved both stability and noise cancellation. Figures 25 and 26 show the response for the Modeling error and the MSE of the intermittent signal, respectively.

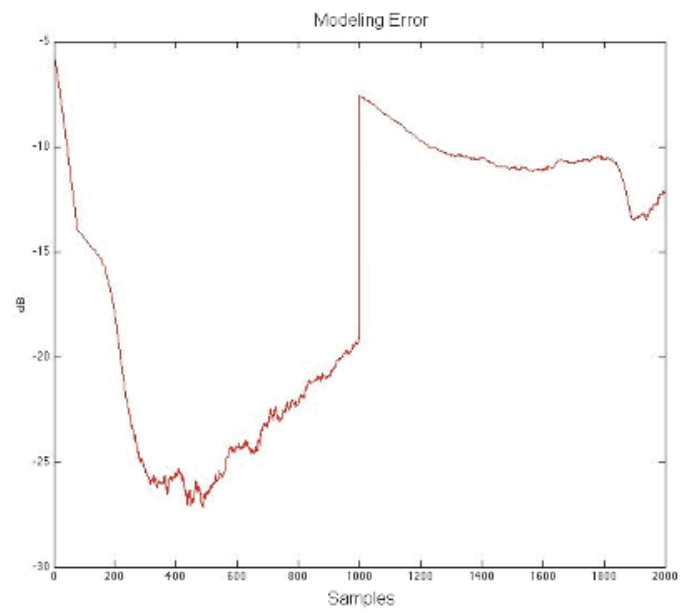

Fig. 25. Relative Modeling Error for Intermittent Signal - Filters with 32 Random Coefficients

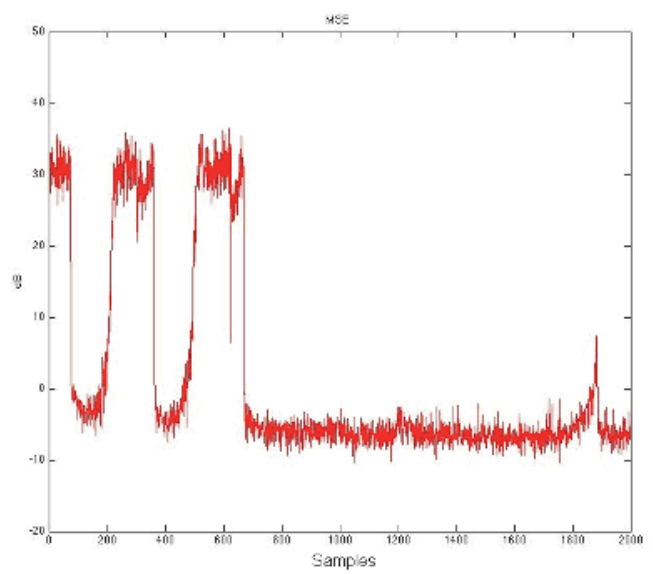

Fig. 26. MSE for Intermittent Signal - Filters with 32 Random Coefficients

Finally, for the impulsive input signal, a similar behavior to the previous test may be observed. The system took a very long time to start converging alter the most abrupt impulse and was not able to cancel noise. 


\subsubsection{Filters with 20 real coefficients}

The last set of tests that were made involved the use of 20th order filters with real coefficients. These coefficients were taken from the work done in (Lopez-Caudana et al, 2009) to determine the values of the primary and secondary path filters for an air duct. Table 4 shows the values used for the feedforward and feedback step sizes, as well as the range of step sizes used for the secondary path filter. The values were set by trial and error, starting with the values that were determined with the previous test.

\begin{tabular}{|c|c|c|}
\hline Signal & $\begin{array}{c}\text { Step size } \\
\mu_{\mathrm{w}}, \mu_{\mathrm{m}}\end{array}$ & $\begin{array}{c}\text { Step size } \\
\mu_{\mathrm{s}}\end{array}$ \\
\hline Continuous & 0.000001 & $0.0001-0.001$ \\
\hline Intermittent & 0.000001 & $0.0001-0.001$ \\
\hline Impulsive & 0.000001 & $0.0001-0.001$ \\
\hline
\end{tabular}

Table 4. Filters Step Size Used in Proposed Analysis

For each of the three cases, a white noise with zero mean and variance equal to 0.05 was used in the system. Since there were not enough resources to implement an abrupt secondary path change (which means there was only one set of values available for the secondary path filter from (Lopez-Caudana et al, 2008)), a gradual change was made, given by the sum of a sinusoidal function to the secondary path coefficients, from iteration 1000 to 1100. Since the best response was shown by the continuous signal, Figure 27 shows the Modeling error for this case, while Figure 28 shows the MSE.

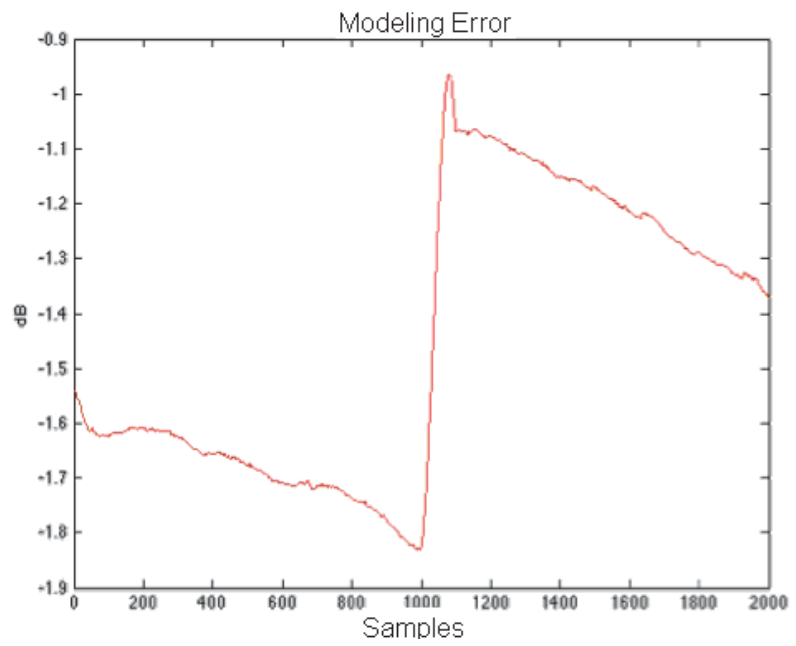

Fig. 27. Relative Modeling Error for Continuous Signal - Filters with 20 Real Coefficients 


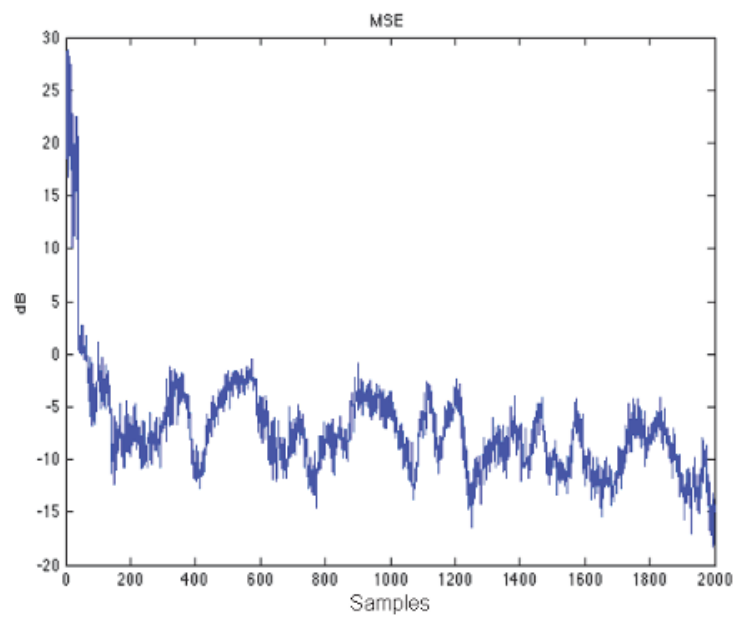

Fig. 28. MSE for Continuous Signal - Filters with 20 Real Coefficients

From Table 4, it is noticeable that the step sizes had to be considerably reduced, about an order of 1000 in comparison to the values established for the tests with 20 random coefficients. This is due to the fact that the coefficient values are not necessarily within a range of -1 to 1 , so the secondary path modeling needs a smaller step size to be able to achieve a point of convergence.

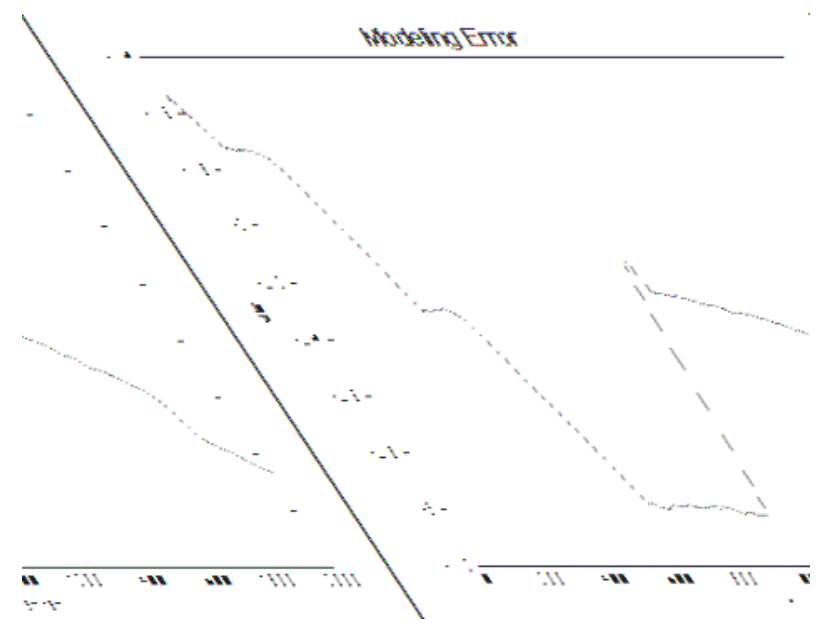

Fig. 29. Relative Modeling Error for Intermittent Signal - Filters with 20 Real Coefficients 


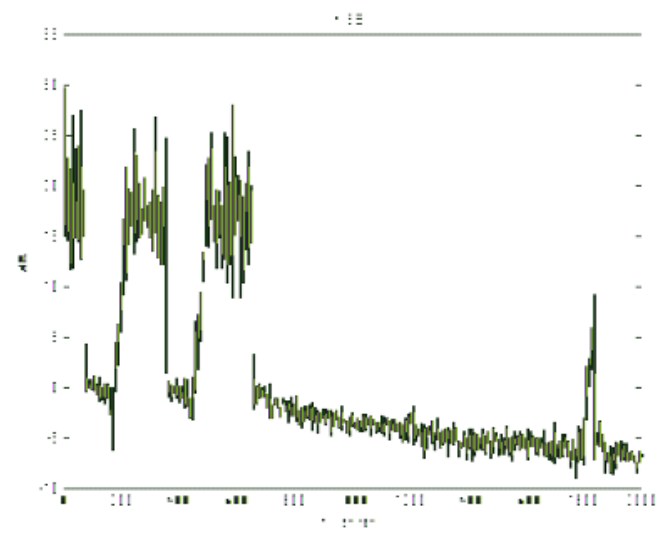

Fig. 30. MSE for Intermittent Signal - Filters with 20 Real Coefficients

According to figure 27 and 28 , the system needed more time to converge to a certain value, compared with the results from the previous tests. This is because the values of the step sizes are very small and cause the convergence process to go slower and, also, the level of noise cancellation to be reduced. For the intermittent signal, the effects of the small step sizes were similar: the system took more time to converge and the level of noise cancellation was reduced. Nonetheless, the response did achieve stability at some point during the simulation. Figure 29 and Figure 30 correspond to the Modeling error and MSE for the intermittent signal, respectively.

However, in the case of the impulsive input signal the results were not as good as they had been expected. Due to the fact that there are very abrupt changes in the signal amplitude, and the step size is very small, there comes a point where the values of the coefficients tend to infinity and the simulation stops at about iteration 200 .

\subsection{An special case: the analysis of hybrid system versus neutralization system 4.3.1 Acoustic feedback path modeling}

It is important to bring attention to the most common way to eliminate acoustic feedback, which is to make an online path modeling, like indicated on (Kuo \& Morgan, 1999) and, more recently, in relevant work like (Akthar et al, 2007). However, one of the main characteristics of the hybrid system presented in (Lopez-Caudana et al, 2008), is that it does not take the secondary path modeling into consideration, but instead takes advantage of the inherent robustness of hybrid systems when it comes to acoustic feedback.

The system in Figure 31, proposed by Kuo in (Kuo, 2002), was used to compare the robustness of the HANC system against the neutralization system.

The details of the system in Figure 7 may be consulted in (Kuo, 2002), however, an important fact of this system is that it uses additive noise for modeling. Also, as mentioned in (Akthar et al, 2007), it has some limitations in reference to predictable noise sources. 


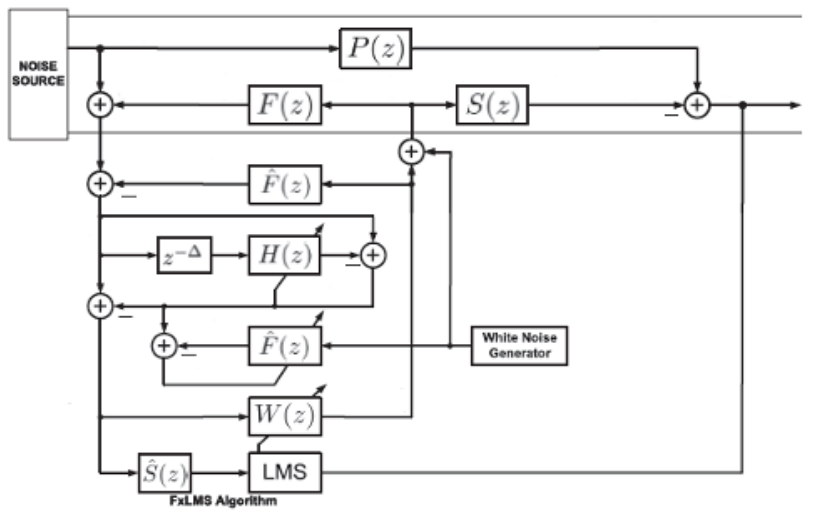

Fig. 31. Kuo's Neutralization System

\subsubsection{Evaluation methodology}

This section shows the simulation of the experiments developed to verify the proposed method. First, we should list the main aspects of the analysis: the experimental test conditions and the types of signals to be cancelled. Three paths were used: the main or primary path $P(z)$, the secondary path $S(z)$, and the acoustic feedback path $F(z)$. All the filters used in the evaluated proposals are finite response filters (FIR), due to their convenient convergence to a minimum value. The values of these paths are taken from (Kuo \& Morgan, 1996), and represent the experimental values of a given duct. However, test were also performed for random values limited by certain coefficients, as done in (Lopez-Caudana et al, 2008), to verify the performance of the systems. A total of 25 coefficients will be used in all paths so as to report an extreme condition for a real duct under analysis; also, lengths of 32, 12, and 22 coefficients, in that order, will be used for the given paths.

Furthermore, six different types of signals were used for the analyzed systems:

a. A sinusoidal reference signal with frequency of $300 \mathrm{~Hz}$, and $30 \mathrm{~dB}$ SNR;

b. A reference signal composed of the sum of narrow band sinusoidal signals of 100, 200, 400 , and $600 \mathrm{~Hz}$;

c. The rest of the reference signals are .wav audio files with recordings of real noise sources, which are "motor", "airplane", "snoring", and "street", as in (Lopez-Caudana et al, 2009).

To initialize $\hat{S}(z)$, the offline secondary path modeling is stopped when the Modeling error has been reduced up to $-35 \mathrm{~dB}$, similar to (Lopez-Caudana et al, 2008). The excitation signal $v(n)$, is white Gaussian noise with variance equal to 0.05 .

The values for the step size are adjusted by trial-and-error to achieve a faster convergence and stability, following the guidelines from previous work on HANC (Lopez-Caudana et al, 2009), and the values selected in (Akthar et al, 2007) for neutralization. A summary of the used values for $\mu$, alter choosing the most convenient parameters, is shown in Table 5 . 


\begin{tabular}{|c|c|c|c|}
\hline System & $\begin{array}{c}\text { Primary } \\
\text { Path } \\
\mu_{\mathrm{P}}\end{array}$ & $\begin{array}{c}\text { Secondary } \\
\text { Path } \\
\mu_{\mathrm{S}}\end{array}$ & $\begin{array}{c}\text { Feedback } \\
\text { Path } \\
\mu_{\mathrm{F}}\end{array}$ \\
\hline $\begin{array}{c}\text { Neutralization } \\
\text { System }\end{array}$ & 0.000001 & 0.00005 & 0.00005 \\
\hline $\begin{array}{c}\text { Hybrid } \\
\text { System }\end{array}$ & 0.001 & 0.001 & \\
\hline
\end{tabular}

Table 5. Filters Step Size Used in Proposed Analysis

\subsubsection{Experimental results}

The performance of the systems is shown next, graphing the mean square error as a typical measurement for these kinds of systems when measuring de power of the error output, in dB (Kuo \& Morgan, 1999). The analysis cases are as follows.

\subsubsection{Analysis with different input signals}

This is the longest case due to the fact that it shows the systems' performance for each mentioned signal. All paths have the same order ( 25 coefficients), taking critical conditions in a real duct for the analyzed phenomenon. Figure 32 to Figure 37 shows the result of the systems analysis with the previously mentioned set of signals. All results are shown in dBs, measuring the error power at the output (Mean Square Error).

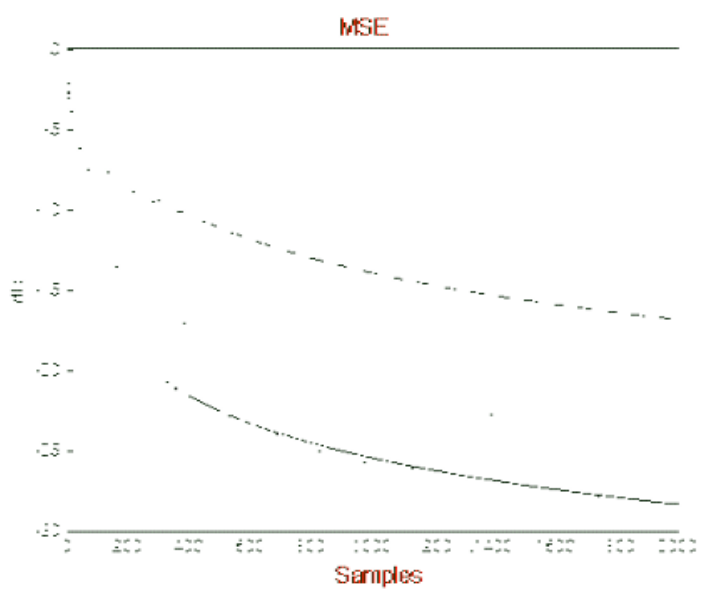

Fig. 32. MSE with "sinusoidal" reference signal: Hybrid System; Neutralization; Feedforward 


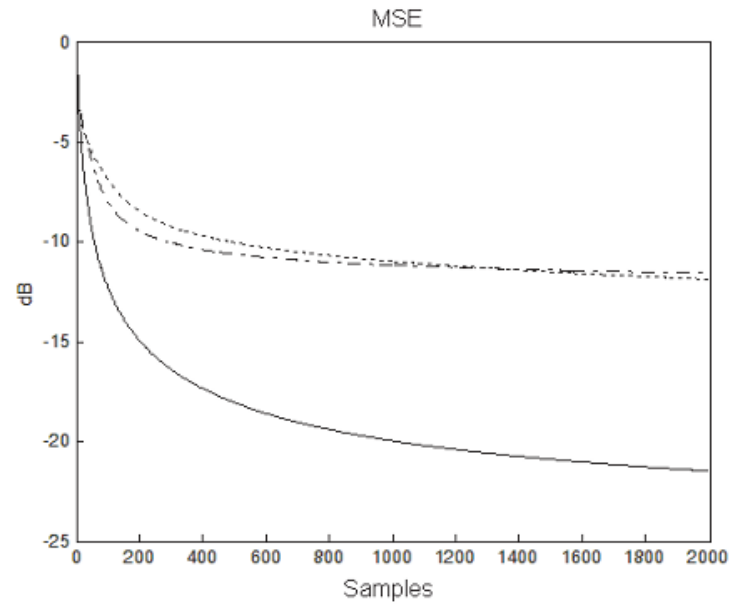

Fig. 33. MSE with "4 tones" reference signal: Hybrid System; Neutralization; Feedforwarard

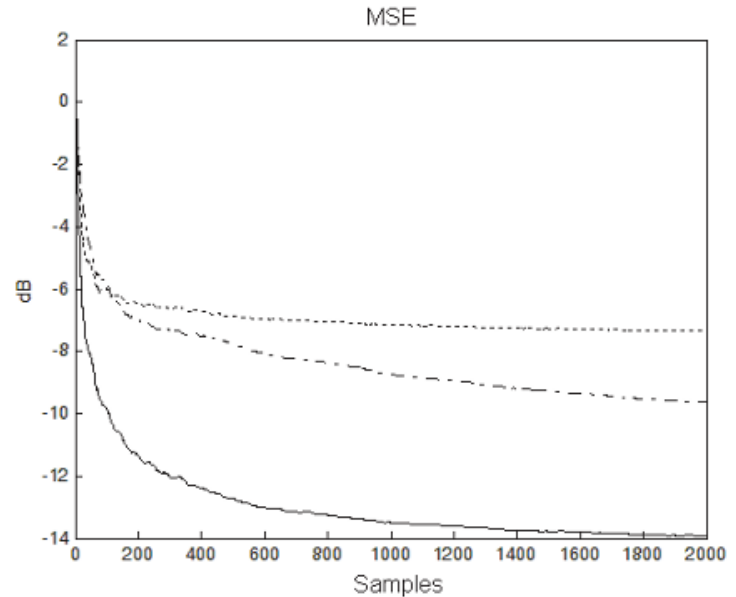

Fig. 34. MSE with "Motor" reference signal: Hybrid System; Neutralization; Feedforward 


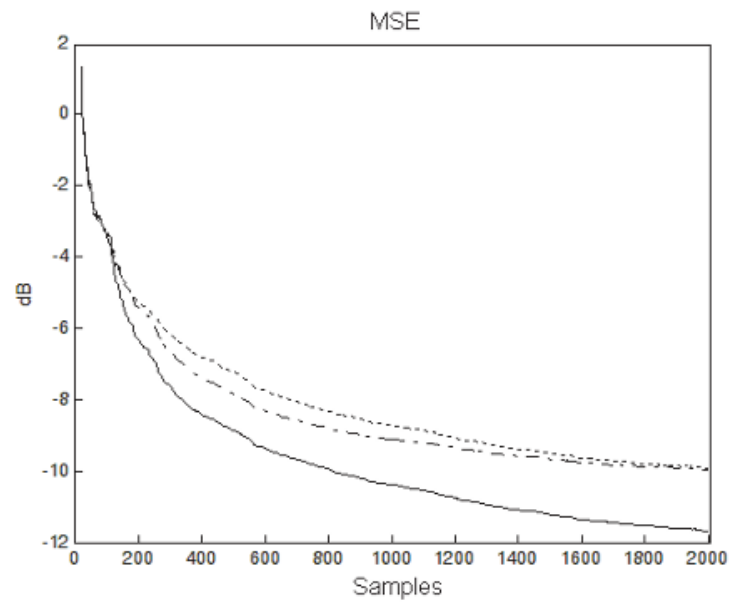

Fig. 35. MSE with "Airplane" reference signal: Hybrid System; Neutralization; Feedforward

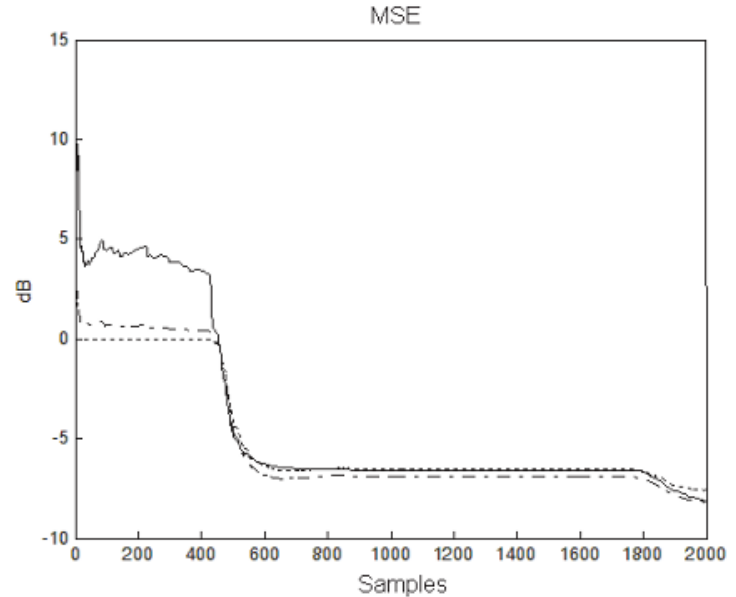

Fig. 36. MSE with “Snoring” reference signal: Hybrid System; Neutralization; Feedforward 


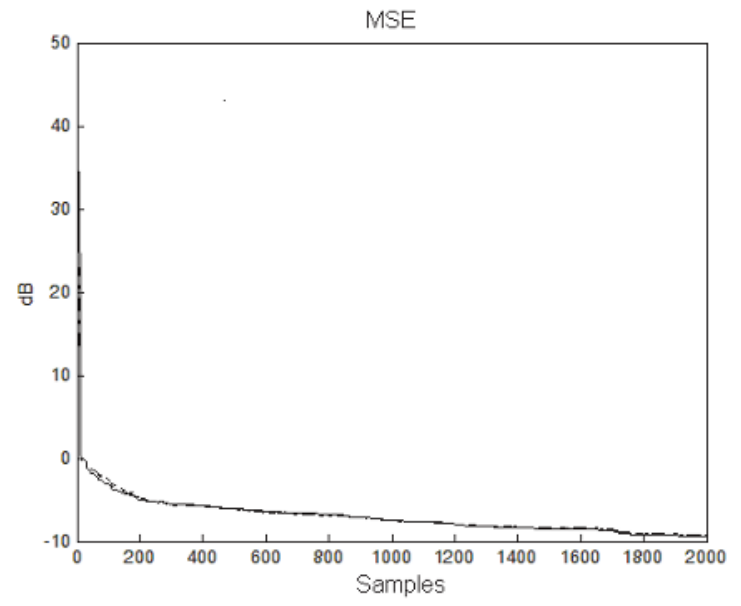

Fig. 37. MSE with "Street" reference signal: Hybrid System; Neutralization; Feedforward 4.3.3.2 Analysis of systems with random path's coefficient values

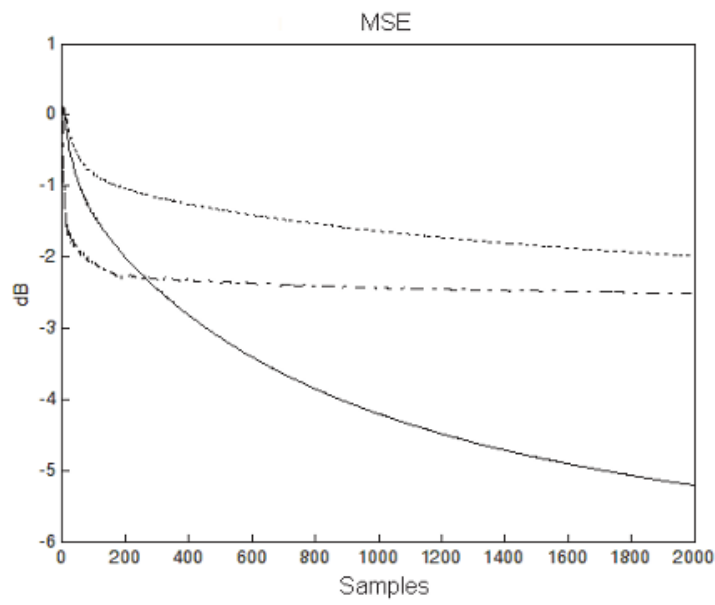

Fig. 38. MSE with “4 tones" reference signal: Hybrid System; Nenutralization; Feedfororward 


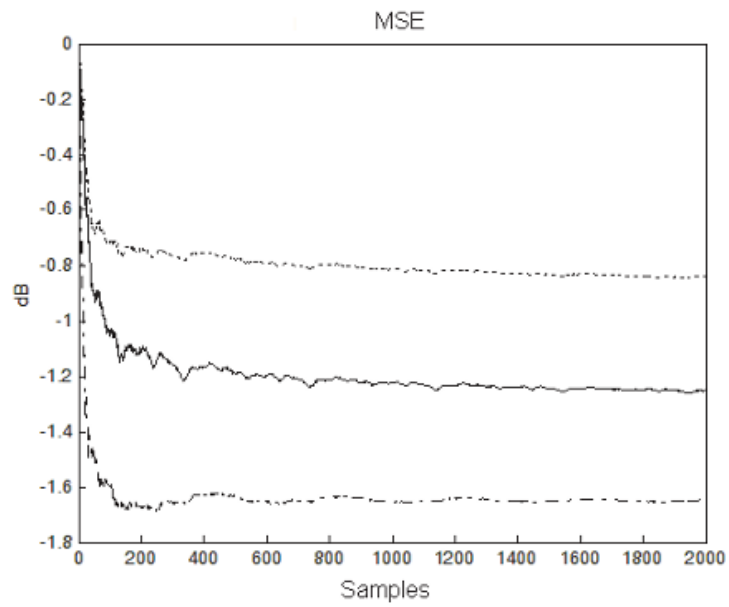

Fig. 39. MSE with "motor" reference signal: Hybrid System; Neutralizationon; Feedfororward

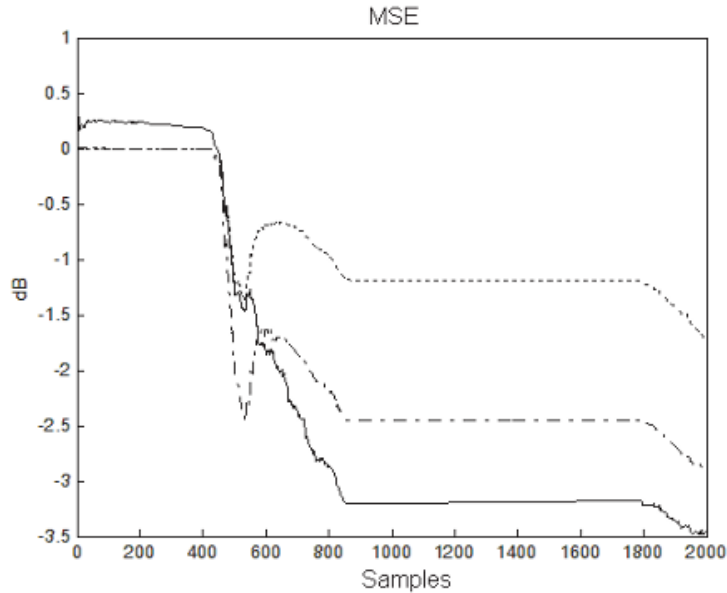

Fig. 40. MSE with "snoring" reference signal: Hybrid System; Neutralization; Feedforward

For this case, the values for the paths proponed by Kuo in (Kuo \& Morgan, 1996), were changed for random values between 0.5 and -0.5 , as done in (Kuo \& Morgan, 1996). These random values generate broader spectra since they do not have such a homogeneous behavior as the experimental values from Kuo (Kuo \& Morgan, 1996). Thus, the same conditions from Case 1 were kept, except for the paths' values. We only show the results of 
three signals, considered the most representative from the set of tests done for this paper: " 4 tones", "motor", and "snoring" (classified as continuous and intermittent signals, as mentioned previously).

\subsubsection{Length of the paths under analysis}

In this section the order of the paths is modified in order to analyze the systems' performance, changing them to 32, 12, and 22 coefficients for the primary, secondary, and feedback path. The values of the paths are the same proponed in (Lopez-Caudana et al, 2009). Only the HANC and neutralization systems are analyzed.

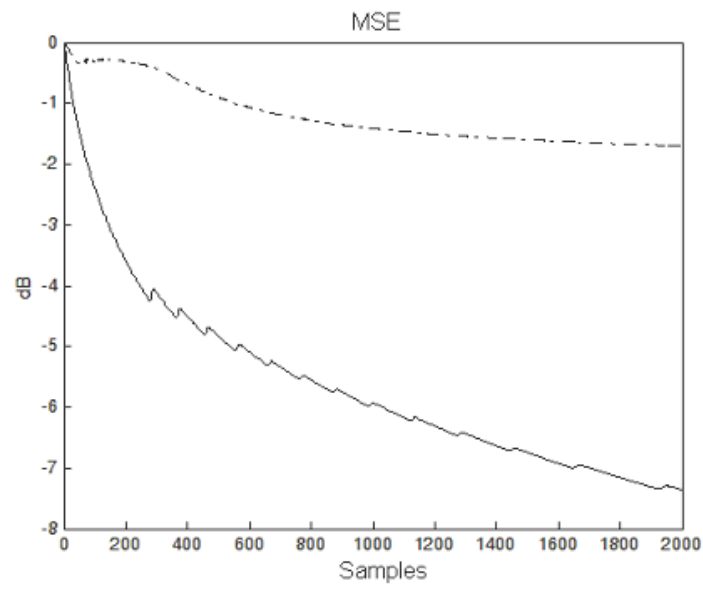

Fig. 41. MSE with " 4 tones" reference signal: $\underline{\text { Hybrid System; Neutralization }}$

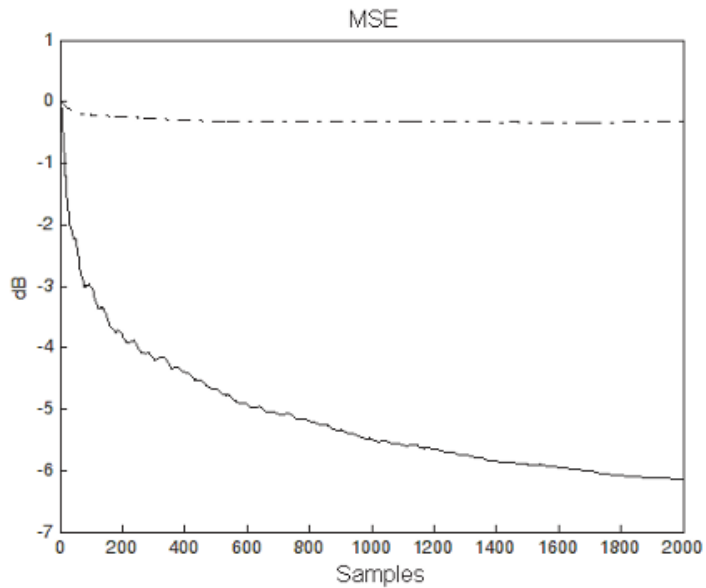

Fig. 42. MSE with "motor" reference signal: Hybrid System; Neutralization 


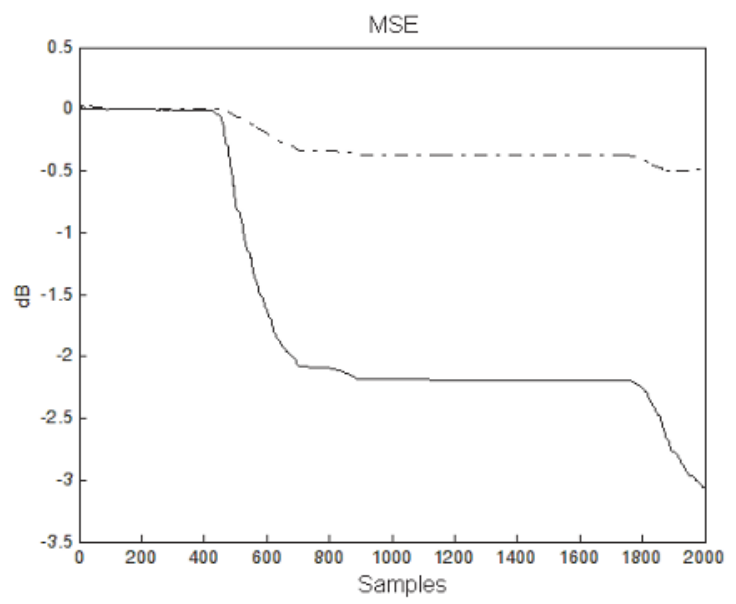

Fig. 43. MSE with "snoring" reference signal: Hybrid System; Neutralization

\subsubsection{Change in secondary path}

An important characteristic of ANC systems is that they must be capable of secondary path online modeling, which is observed in the graphs 44 . There is an abrupt secondary path change in the thousandth iteration - taken from (Lopez-Caudana et al, 2008)- which does not causes the behavior of either system to destabilize when the values for a new secondary path appear.

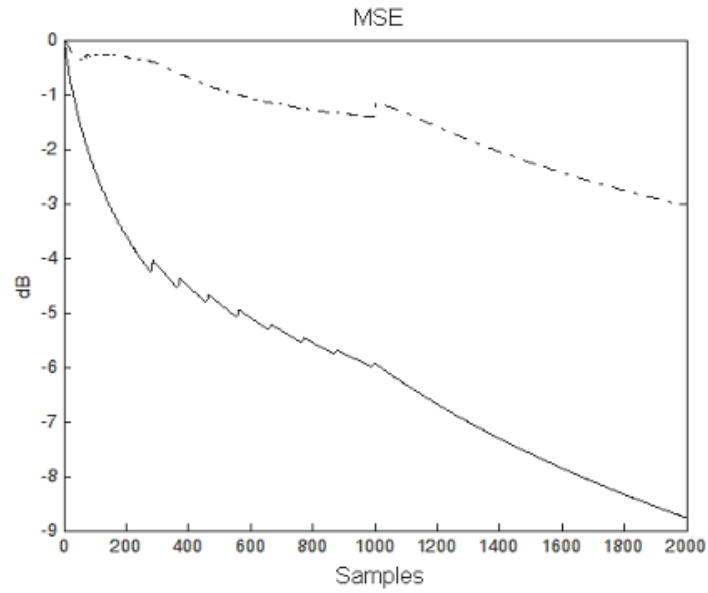

Fig. 44. MSE with "4 tones" reference signal: Hybrid System; Neutralization 


\section{Conclusions}

In this chapter, we have showed a new system with a robustness structure that combines both the Feedforward and Feedback ANC basics systems to improve the frequency range of work. Additionally the consideration of the acoustic feedback and the online secondary path modeling allows the system to be adjustable for any kind of secondary path change (gradual ideally). The computer simulations demonstrate proposed system gives improved performance, at somewhat increased computational cost because the Akhtar's online secondary path method, but this method compensates the noise control process for the Feedforward and Feedback stages.

In the noise control process, two adaptive filters are incorporated with the FXLMS algorithm, one for identification (Feedforward) and other for prediction (Feedback). Both of them achieve a signal that is the result of the noise control process. A VSS-LMS algorithm is used in the secondary path modeling to achieve the fastest optimal solution of the modeling filter. Finally computer simulation results shown support these conclusions and demonstrate the effectiveness of the proposed system.

By other hand, this work successfully showed a set of analysis parameters to characterize the performance of a hybrid ANC system with acoustic feedback and the online secondary path estimation, outsider of an ideal simulation environment.

Different parameters were considered, such as the order of the paths, and the abrupt secondary path change, to achieve a fuller analysis. Also, the hybrid systems was subject not only to those reference signals considered 'classical', but to a set of real noise recordings, which allowed to analyze more complete characteristics from a system, while making it real. In the process of noise control, two adaptive filters were incorporated with the FXLMS algorithm, one for identification (Feedforward), and one for prediction (Feedback). Even though an adaptive filter for the acoustic feedback path was not considered, as done for systems designed to specifically attack this problem, the performance of the hybrid system is more efficient, compared to a system that is set out to solve that particular problem. Also, the, the computer simulation results show the support for these conclusions and confirm the effectiveness of the proposed system.

Based on the presented classification of noise signals we can conclude that:

1. The system has an efficient response to continuous signals, showing a stable response as well as considerable noise cancellation;

2. The response to intermittent signals is acceptable in general, though it depends to a certain extent on the waveform of the input noise signal. However, the system converges to a certain value and is able to cancel noise, though less than in the case of the continuous signals; and

3. In reference to impulsive signals, the system achieves a convergent point slowly, but cannot cancel noise significantly because the change in the signal amplitude is too abrupt for the system to adapt the filter coefficients efficiently enough.

When tested with real-value filter coefficients, the system presented a decrease on its convergence speed, which affected the performance of the system mainly in the case of an impulsive input noise signal. Finally, it must be considered that the results presented for a real-value filter coefficients refer to only one specific kind of duct. This means that the response could probably improve in a different environment or in a duct with different properties. This presents a problem for the designer of a hybrid ANC system, as it means that, for each environment where the system is to be implemented; there would be the need 
to accurately individualize the parameters to achieve the desired response. However difficult, this may not be impossible to do, so there is still a lot of work to be done with hybrid ANC systems.

\section{Acknowledgement}

The contributions of several students from Communications and Electronic Engineering from Tecnologico de Monterrey, Mexico City Campus, are gratefully acknowledged and the guidance from Dr. Hector Perez-Meana from IPN SEPI ESIME CULHUACAN. This work has been supported by Mechatronic's Department of the Engineering and Architecture School from Tecnologico de Monterrey, Mexico City Campus.

\section{References}

Akthar Muhammad Tahir, Masahide Abe, and Masayuki Kawamata (2004). "Modifiedfiltered-x LMS algorithm based active noise control system with improved online secondary-path modeling" in Proc. IEEE 2004 Int. Mid. Symp. Circuits Systems (MWSCAS2004), Hiroshima, Japan, Jul. 25-28, 2004, pp. I-13-I-16, 2004.

Akhtar, M.T.; Abe, M.; Kawamata, M., (2006). “A new variable step size LMS algorithmbased method for improved online secondary path modeling in active noise control systems". IEEE Transactions on Audio, Speech, and Language Processing, Volume 14, Issue 2, March 2006 Page(s):720 - 726.

Akhtar et al. (2007) Muhammad Tahir Akhtar, M. Tufail, Masahide Abe, y Masayuki Kawamata. "Acoustic feedback neutralization in active noise control systems" IEICE Electronics Express. Vol. 4, No. 7, pp. 221 - 226.

Akhtar et al. (2007) Muhammad Tahir Akhtar, Masahide Abe, y Masayuki Kawamata. "On active Noise Control Systems with Online Acoustic Feedback Path Modeling" in IEEE Transactions on Audio, Speech, and Language Processing, Vol. 15, No. 2, February 2007 pp. 593-599.

Eriksson L. J., Allie M. C., y Bremigan C. D. (1998), Active noise control using adaptive digital signal processing, Proc. ICASSP, , pp. 2594-2597.

Kuo Sen M, Dennis R. Morgan (1999). "Active Noise Control Systems: A tutorial review" Proc. IEEE, vol. 87, no. 6, pp. 943-973, Junio 1999.

Kuo Sen M, Dennis R. Morgan (1996). "Active Noise Control Systems: Algorithms and DSP Implementations" New York: Wiley Series in Telecommunications and Signal Processing Editors, 1996.

Kuo Sen M, (2002) "Active Noise Control System and Method for On-Line Feedback Path Modeling" US Patent 6,418,227, Julio 9, 2002.

Lopez-Caudana Edgar, Pablo Betancourt, Enrique Cruz, Mariko Nakano Miyatake, Hector Perez-Meana (2008). "A Hybrid Active Noise Canceling Structure", International Journal of Circuits, Systems and Signal Processing. Issue 2 Vol 2, 2008. pp 340-346.

Lopez-Caudana Edgar, Pablo Betancourt, Enrique Cruz, Mariko Nakano Miyatake, Hector Perez-Meana (2008) "A Hybrid Noise Cancelling Algorithm with Secondary Path Estimation" WSEAS TRANSACTIONS on SIGNAL PROCESSING Issue 12, Volume 4, December 2008.

Lopez-Caudana, E.; Betancourt, P.; Cruz, E.; Nakano-Miyatake, M.; Perez-Meana, H., (2008). "A hybrid active noise cancelling with secondary path modeling", Circuits and 
Systems, 2008. MWSCAS 2008. 51st Midwest Symposium on . 10-13 Aug. 2008 Page(s):277 - 280.

Lopez-Caudana Edgar, Paula Colunga, Alejandro Celis, Maria J. Lopez, and Hector PerezMeana (2009). "Evaluation of a Hybrid ANC System with Acoustic Feedback and Online Secondary Path Modeling". 19th International Conference on Electronics, Communications and Computers 2009, Cholula, Puebla. 26-28 Febrero de 2009.

Lopez-Caudana, Edgar, Paula Colunga, Rogelio Bustamante and Hector Perez-Meana, (2010)."Evaluation for a Hybrid Active Noise Control System with Acoustic Feedback". 53rd IEEE Int'l Midwest Symposium on Circuits E Systems, tSeattle, Washington from August 1-4, 2010.

Nakano M., H. Perez (1995), A Time Varying Step Size Normalized LMS Algorithm for Adaptive Echo Canceler Structure, IEICE Trans. on Fundamentals of Electronics Computer Sciences, Vol. E-78-A, 1995, pp. 254-258.

Romero, A; Perez-Meana, H.; Lopez-Caudana, E. (2008); “A Hybrid Active Noise Canceling Structure", International Journal of Circuits, Systems and Signal Processing. Issue 2 Vol 2, 2008. pp 340-346.

Romero, Nakano-Miyatake, Perez-Meana (2008), A Hybrid Noise Canceling Structure with Secondary Path Estimation, WSEAS Recent Advances in Systems, Communications and Computers, 2008, pp.194-199.

http://www.freesfx.co.uk/soundeffectcats.html

Free Sound Effects, Samples \& Music. Free Sound Effects Categories. Visited on July 3, 2008.

http://www.nonoise.org/library/envnoise/index.htm

Brüel \& Kjær Sound \& Vibration Measurement A/S. Environmental Noise Booklet. Visited on March 25, 2008. 


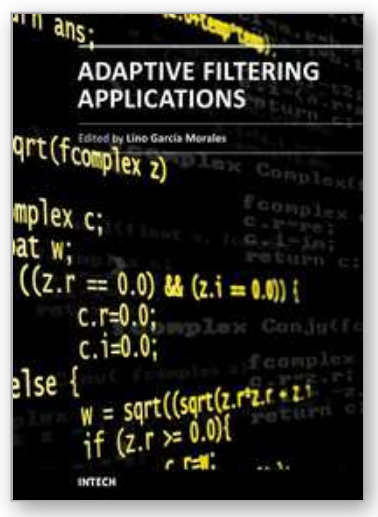

\author{
Adaptive Filtering Applications \\ Edited by Dr Lino Garcia
}

ISBN 978-953-307-306-4

Hard cover, 400 pages

Publisher InTech

Published online 24, June, 2011

Published in print edition June, 2011

Adaptive filtering is useful in any application where the signals or the modeled system vary over time. The configuration of the system and, in particular, the position where the adaptive processor is placed generate different areas or application fields such as: prediction, system identification and modeling, equalization, cancellation of interference, etc. which are very important in many disciplines such as control systems, communications, signal processing, acoustics, voice, sound and image, etc. The book consists of noise and echo cancellation, medical applications, communications systems and others hardly joined by their heterogeneity. Each application is a case study with rigor that shows weakness/strength of the method used, assesses its suitability and suggests new forms and areas of use. The problems are becoming increasingly complex and applications must be adapted to solve them. The adaptive filters have proven to be useful in these environments of multiple input/output, variant-time behaviors, and long and complex transfer functions effectively, but fundamentally they still have to evolve. This book is a demonstration of this and a small illustration of everything that is to come.

\title{
How to reference
}

In order to correctly reference this scholarly work, feel free to copy and paste the following:

Edgar Omar Lopez-Caudana (2011). Active Noise Cancellation: The Unwanted Signal and the Hybrid Solution, Adaptive Filtering Applications, Dr Lino Garcia (Ed.), ISBN: 978-953-307-306-4, InTech, Available from: http://www.intechopen.com/books/adaptive-filtering-applications/active-noise-cancellation-the-unwantedsignal-and-the-hybrid-solution

\section{INTECH}

open science | open minds

\section{InTech Europe}

University Campus STeP Ri

Slavka Krautzeka 83/A

51000 Rijeka, Croatia

Phone: +385 (51) 770447

Fax: +385 (51) 686166

www.intechopen.com

\section{InTech China}

Unit 405, Office Block, Hotel Equatorial Shanghai

No.65, Yan An Road (West), Shanghai, 200040, China 中国上海市延安西路65号上海国际贵都大饭店办公楼 405 单元

Phone: +86-21-62489820

Fax: +86-21-62489821 
(C) 2011 The Author(s). Licensee IntechOpen. This chapter is distributed under the terms of the Creative Commons Attribution-NonCommercialShareAlike-3.0 License, which permits use, distribution and reproduction for non-commercial purposes, provided the original is properly cited and derivative works building on this content are distributed under the same license. 